\title{
EXISTENCE AND UNIQUENESS OF GLOBAL WEAK SOLUTION TO A KINETIC MODEL FOR THE SEDIMENTATION OF ROD-LIKE PARTICLES*
}

\author{
XIUQING $\mathrm{CHEN}^{\dagger}$, XIAOLONG $\mathrm{LI}^{\ddagger}$, AND JIAN-GUO LIU
}

\begin{abstract}
We investigate a kinetic model for the sedimentation of dilute suspensions of rod-like particles under gravity, deduced by Helzel, Otto, and Tzavaras (2011), which couples the impressible (Navier-)Stokes equation with the Fokker-Planck equation. With a no-flux boundary condition for the distribution function, we establish the existence and uniqueness of a global weak solution to the two dimensional model involving the Stokes equation.
\end{abstract}

Key words. Stokes equation, Fokker-Planck equation, global weak solution, uniqueness.

AMS subject classifications. 35Q30, 35K55, 76D05, 65M06.

\section{Introduction}

Rod-like particle suspensions in fluid are common in nature, including examples such as bacteria swimming in the water and liquid crystal molecules moving in a solvent. The dilute suspensions of passive rod-like particles can be effectively modeled by a coupled microscopic Fokker-Planck equation and macroscopic (Navier-)Stokes equation, known as the Doi model (see Doi [9]; Doi and Edwards [10]). We refer to [23] for the Doi model for suspensions of active rod-like particles without considering the effects of gravity. Recently an extended model under gravity was introduced by Hezel, Otto, and Tzavaras [16], which reads

$$
\begin{aligned}
\partial_{t} f+\nabla_{\mathbf{x}} \cdot(\mathbf{u} f)-\Delta_{\mathbf{n}} f+\nabla_{\mathbf{n}} \cdot\left[(\operatorname{Id}-\mathbf{n} \otimes \mathbf{n}) \nabla_{\mathbf{x}} \mathbf{u n} f\right] & =\begin{array}{r}
\nabla_{\mathbf{x}} \cdot\left[(\operatorname{Id}+\mathbf{n} \otimes \mathbf{n}) \mathbf{e}_{2} f\right] \\
+\gamma \nabla_{\mathbf{x}} \cdot\left[(\operatorname{Id}+\mathbf{n} \otimes \mathbf{n}) \nabla_{\mathbf{x}} f\right]
\end{array} \\
\sigma & =\int_{\mathbb{S}^{d-1}}[(d \mathbf{n} \otimes \mathbf{n}-\mathrm{Id}) f] d \mathbf{n}, \\
\operatorname{Re}\left[\partial_{t} \mathbf{u}+\left(\mathbf{u} \cdot \nabla_{\mathbf{x}}\right) \mathbf{u}\right]-\Delta_{\mathbf{x}} \mathbf{u}+\nabla_{\mathbf{x}} p=\beta \gamma \nabla_{\mathbf{x}} \cdot \sigma-\beta\left(\int_{\mathbb{S}^{d-1}} f d \mathbf{n}\right) \mathbf{e}_{2} & \\
\nabla_{\mathbf{x}} \cdot \mathbf{u} & =0
\end{aligned}
$$

where $(t, \mathbf{x}, \mathbf{n}) \in[0, \infty) \times \Omega \times \mathbb{S}^{d-1}, \Omega \subset \mathbb{R}^{d}$ is a bounded domain with $\partial \Omega$ of class $C^{1}$ and $\mathbb{S}^{d-1} \subset \mathbb{R}^{d}$ is the unit sphere; $\sigma$ is a stress tensor, $p$ is the pressure, $\mathbf{e}_{2}$ is the unit vector

*Received: April 20, 2013; accepted (in revised form): July 7, 2013. Communicated by Tiejun Li. The first author acknowledges support from the National Science Foundation of China (grant 11101049), the Research Fund for the Doctoral Program of Higher Education of China (grant 20090005120009). The third author acknowledges support from NSF RNMS (KI-Net) grant \#1107444.

${ }^{\dagger}$ School of Sciences, Beijing University of Posts and Telecommunications, Beijing, 100876, P.R. China; Department of Physics and Department of Mathematics, Duke University, Durham, NC 27708, USA (buptxchen@yahoo.com).

${ }^{\ddagger}$ School of Sciences, Beijing University of Posts and Telecommunications, Beijing, 100876, P.R. China (ttlixiaolong@gmail.com).

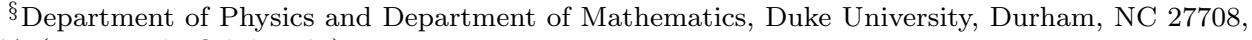
USA (jian-guo.liu@duke.edu). 
in the upward direction; $\nabla_{\mathbf{n}}$ and $\Delta_{\mathbf{n}}$ denote the tangential divergence and LaplaceBeltrami operator on $\mathbb{S}^{d-1}$, respectively. In this model, $f(t, \mathbf{x}, \mathbf{n})$ is a distribution function which represents the configuration of a suspension of rod-like particles and $\mathbf{u}(t, \mathbf{x})$ is the fluid velocity induced by the other particles in the suspension.

The coefficients $\operatorname{Re} \geq 0, \beta>0$, and $\gamma>0$ are constants. Re is a Reynolds number; $\beta$ measures the relation between the rate of work of buoyancy vs. the rate of work of the viscous force; $\gamma$ measures the relation between the rate of work of elastic forces vs. the rate of work of buoyancy (see [16], Remark 2.1-2.2). For convenience, if $R e=0$, the model including a Stokes equation is called Stokes-type.

The first term on the right hand side of (1.1) represents the effects of gravity on rod-like particles. Taking this particular form are due to the fact that the frictional coefficient in the tangential direction is twice as large as that in the normal direction. This result comes from classical slender body theory (see $[17,4,19]$ ). Since horizontally orientated particles sediment slower than particles with a vertical orientation, particles move sideways with a slight concentration in 45 degrees. One of the most peculiar phenomena in sedimentation of rod-like particles is packet formation and alignment in the gravity direction (see [16]). An anisotropic diffusivity in the second term on the right hand side of (1.1) is also due to the inhomogeneity of the frictional coefficients in the tangential direction and normal direction.

Defining the concentration density $\rho:=\int_{\mathbb{S}^{d-1}} f d \mathbf{n}$ and integrating (1.1) over $\mathbb{S}^{d-1}$, we deduce that

$$
\partial_{t} \rho+\mathbf{u} \cdot \nabla_{\mathbf{x}} \rho=\nabla_{\mathbf{x}} \cdot\left(\mathbf{e}_{2} \rho+\gamma \nabla_{\mathbf{x}} \rho+\int_{\mathbb{S}^{d-1}} \mathbf{n} \otimes \mathbf{n} f d \mathbf{n} \mathbf{e}_{2}+\gamma \nabla_{\mathbf{x}} \cdot \int_{\mathbb{S}^{d-1}} \mathbf{n} \otimes \mathbf{n} f d \mathbf{n}\right) .
$$

The last two terms account for the anisotropic effects of gravity and diffusivity. As a result, we do not have $L^{\infty}$ and $L^{2}$ estimates for density $\rho$.

In contrast to the FENE and the Hookean models with centre-of-mass diffusion, the density $\rho$ satisfies a convection diffusion equation

$$
\partial_{t} \rho+\mathbf{u} \cdot \nabla_{\mathbf{x}} \rho-D \Delta_{\mathbf{x}} \rho=0,
$$

and where the maximum principle holds (see [2]-[3]), in the Doi model for active rod-like particle suspensions (see [5]),

$$
\partial_{t} \rho+\mathbf{u} \cdot \nabla_{\mathbf{x}} \rho+\nabla_{\mathbf{x}} \cdot\left(\int_{\mathbb{S}^{d-1}}(\alpha \mathbf{n} f) d \mathbf{n}-D \nabla_{\mathbf{x}} \rho\right)=0,
$$

where $\rho$ has $L^{2}$-estimate. The $L^{\infty}$ or $L^{2}$-estimate for $\rho$ is the foundation for establishing the global entropy weak solution in [2]-[3] and [5].

It can be shown that this model with boundary conditions

$$
\left.(\operatorname{Id}+\mathbf{n} \otimes \mathbf{n})\left(\mathbf{e}_{2} f+\gamma \nabla_{\mathbf{x}} f\right) \cdot \boldsymbol{v}\right|_{\partial \Omega}=0,\left.\quad \mathbf{u}\right|_{\partial \Omega}=0,
$$

has the following entropy estimate (see [16]):

$$
\begin{aligned}
& \frac{d}{d t} \int_{\Omega}\left(\beta \int_{\mathbb{S}^{d-1}}\left(\gamma f \ln f-\gamma f+f \mathbf{x} \cdot \mathbf{e}_{2}\right) d \mathbf{n}+\frac{\operatorname{Re}}{2}|\mathbf{u}|^{2}\right) d \mathbf{x} \\
& +4 \beta \gamma \int_{\Omega \times \mathbb{S}^{d-1}}\left|\nabla_{\mathbf{n}} \sqrt{f}\right|^{2} d \mathbf{n} d \mathbf{x}+\int_{\Omega}\left|\nabla_{\mathbf{x}} \mathbf{u}\right|^{2} d \mathbf{x} \\
& +\beta \int_{\Omega \times \mathbb{S}^{d-1}}(\mathbf{I}+\mathbf{n} \otimes \mathbf{n})\left(\mathbf{e}_{2} f+\nabla_{\mathbf{x}} f\right) \cdot\left(\mathbf{e}_{2} f+\nabla_{\mathbf{x}} f\right) \frac{1}{f} d \mathbf{n} d \mathbf{x}=0,
\end{aligned}
$$


which reveals that the total energy

$$
E(\mathbf{u}, f):=\int_{\Omega}\left(\beta \int_{\mathbb{S}^{d-1}}\left(\gamma f \ln f-\gamma f+f \mathbf{x} \cdot \mathbf{e}_{2}\right) d \mathbf{n}+\frac{\operatorname{Re}}{2}|\mathbf{u}|^{2}\right) d \mathbf{x}
$$

is dissipated.

For this model, without an $L^{\infty}$ or $L^{2}$-estimate of the density $\rho$, the entropy estimate is not enough to establish the existence of global weak solutions. We also need $L^{2}$-estimates for the distribution $f$. In fact, multiplying (1.1) by $f$ and integrating on $\Omega \times \mathbb{S}^{d-1}$, one has

$$
\begin{aligned}
& \frac{1}{2} \frac{d}{d t} \int_{\Omega \times \mathbb{S}^{d-1}}|f|^{2} d \mathbf{n} d \mathbf{x}+\int_{\Omega \times \mathbb{S}^{d-1}}\left|\nabla_{\mathbf{n}} f\right|^{2} d \mathbf{n} d \mathbf{x}+\gamma \int_{\Omega \times \mathbb{S}^{d-1}}(\mathrm{Id}+\mathbf{n} \otimes \mathbf{n}) \nabla_{\mathbf{x}} f \cdot \nabla_{\mathbf{x}} f d \mathbf{n} d \mathbf{x} \\
= & -\int_{\Omega \times \mathbb{S}^{d-1}}(\mathrm{Id}+\mathbf{n} \otimes \mathbf{n}) \mathbf{e}_{2} f \cdot \nabla_{\mathbf{x}} f d \mathbf{n} d \mathbf{x}+\frac{1}{2} \int_{\Omega \times \mathbb{S}^{d-1}}\left[(\mathrm{Id}-\mathbf{n} \otimes \mathbf{n}) \nabla_{\mathbf{x}} \mathbf{u n}\right] \cdot \nabla_{\mathbf{n}} f^{2} d \mathbf{n} d \mathbf{x} .
\end{aligned}
$$

Because Id $+\mathbf{n} \otimes \mathbf{n}$ is a positive definite matrix with the smallest eigenvalue 1, we have

$$
\left|\nabla_{\mathbf{x}} f\right|^{2} \leq(\operatorname{Id}+\mathbf{n} \otimes \mathbf{n}) \nabla_{\mathbf{x}} f \cdot \nabla_{\mathbf{x}} f .
$$

This and Cauchy-Schwartz inequality yield that

$$
\begin{aligned}
& \frac{1}{2} \frac{d}{d t} \int_{\Omega \times \mathbb{S}^{d-1}}|f|^{2} d \mathbf{n} d \mathbf{x}+\int_{\Omega \times \mathbb{S}^{d-1}}\left[\left|\nabla_{\mathbf{n}} f\right|^{2}+\frac{\gamma}{2}\left|\nabla_{\mathbf{x}} f\right|^{2}\right] d \mathbf{n} d \mathbf{x} \\
\leq & C \int_{\Omega \times \mathbb{S}^{d-1}}|f|^{2} d \mathbf{n} d \mathbf{x}+\frac{1}{2} \int_{\Omega \times \mathbb{S}^{d-1}}\left[(\operatorname{Id}-\mathbf{n} \otimes \mathbf{n}) \nabla_{\mathbf{x}} \mathbf{u n}\right] \cdot \nabla_{\mathbf{n}} f^{2} d \mathbf{n} d \mathbf{x} .
\end{aligned}
$$

Integrating by parts, we have

$$
\begin{aligned}
I & =: \int_{\Omega \times \mathbb{S}^{d-1}}\left[(\mathrm{Id}-\mathbf{n} \otimes \mathbf{n}) \nabla_{\mathbf{x}} \mathbf{u n}\right] \cdot \nabla_{\mathbf{n}} f^{2} d \mathbf{n} d \mathbf{x} \\
& =\int_{\Omega \times \mathbb{S}^{d-1}}(d \mathbf{n} \otimes \mathbf{n}-\mathrm{Id}) f^{2}: \nabla_{\mathbf{x}} \mathbf{u} d \mathbf{n} d \mathbf{x} \leq C\left\|\nabla_{\mathbf{x}} \mathbf{u}\right\|_{L^{2}(\Omega)}\|f\|_{L^{4}\left(\Omega ; L^{2}\left(\mathbb{S}^{d-1}\right)\right)}^{2} .
\end{aligned}
$$

If $d=2$, we have from Gagliardo-Nirenberg and Cauchy-Schwartz inequalities (see (3.42)-(3.43)) that

$$
I \leq \frac{\gamma}{4} \int_{\Omega \times \mathbb{S}^{d-1}}\left|\nabla_{\mathbf{x}} f\right|^{2} d \mathbf{n} d \mathbf{x}+C\left(\left\|\nabla_{\mathbf{x}} \mathbf{u}\right\|_{L^{2}(\Omega)}^{2}+1\right)\|f\|_{L^{2}\left(\Omega \times \mathbb{S}^{d-1}\right)}^{2} .
$$

Therefore,

$$
\begin{aligned}
& \quad \frac{d}{d t} \frac{1}{2} \int_{\Omega \times \mathbb{S}^{d-1}}|f|^{2} d \mathbf{n} d \mathbf{x}+\int_{\Omega \times \mathbb{S}^{d-1}}\left(\left|\nabla_{\mathbf{n}} f\right|^{2}+\frac{\gamma}{4}\left|\nabla_{\mathbf{x}} f\right|^{2}\right) d \mathbf{n} d \mathbf{x} \\
& \leq C\left(\left\|\nabla_{\mathbf{x}} \mathbf{u}\right\|_{L^{2}(\Omega)}^{2}+1\right)\|f\|_{L^{2}\left(\Omega \times \mathbb{S}^{d-1}\right)}^{2} .
\end{aligned}
$$

It follows from (1.13), Gronwall's inequality, and the entropy estimate (1.7) that

$$
\|f\|_{L^{2}\left(\Omega \times \mathbb{S}^{d-1}\right)}^{2} \leq C e^{\int_{0}^{t}\left(\left\|\nabla_{\mathbf{x}} \mathbf{u}\right\|_{L^{2}(\Omega)}^{2}+1\right) d s} \leq C(t) .
$$


For $d \geq 3$, we cannot perform estimates similar to (1.12)-(1.14).

With the entropy estimate and two dimensional (2D) $L^{2}$-estimate at hand, in this paper we aim to prove the existence of global weak solutions to the 2D Stokes-type model with boundary conditions (1.6) and initial condition

$$
\left.f\right|_{t=0}=f_{0} \quad \text { on } \Omega \times \mathbb{S}^{d-1} .
$$

There are many works on the mathematical analysis of the Doi model for passive particle suspensions (see $[1,6,7,8,15,16,20,21,25]$ ), in which the density $\rho$ satisfies a transport equation with and without diffusion, and hence the maximum principle holds. In the Doi model for active particle suspensions (see [5]), the density $\rho$ has an $L^{2}$-estimate. The estimates of $\rho$ constitute a common foundation for their proofs. However, this model lacks good estimates for $\rho$ as mentioned before, which is the main difference with other Doi related models. A combination of an entropy estimate and an $L^{2}$-estimate solves this problem.

The paper is organized as follows. Section 2 collects some preliminaries. In Section 3, we prove the existence of the global weak solution for the 2D Stokes-type model, where a semi-implicit scheme is used to construct the approximate problem and compactness is shown. Then in Section 4, we prove the uniqueness of the weak solution.

For conciseness in presentation, we set $\beta=\gamma=1$ in the rest of this paper.

\section{Preliminaries}

The following notations will be used in this paper:

$$
\begin{gathered}
\boldsymbol{L}^{p}(\Omega)=L^{p}\left(\Omega, \mathbb{R}^{d}\right), \boldsymbol{H}^{m}(\Omega)=H^{m}\left(\Omega, \mathbb{R}^{d}\right) \\
\boldsymbol{C}_{0}^{\infty}(\Omega)=C_{0}^{\infty}\left(\Omega, \mathbb{R}^{d}\right), \mathscr{V}=\left\{\mathbf{u} \in \boldsymbol{C}_{0}^{\infty}(\Omega): \nabla_{\mathbf{x}} \cdot \mathbf{u}=0\right\}, \\
\boldsymbol{H}=\left\{\mathbf{u} \in \boldsymbol{L}^{2}(\Omega): \nabla_{\mathbf{x}} \cdot \mathbf{u}=0,\left.\mathbf{u} \cdot \boldsymbol{v}\right|_{\partial \Omega}=0\right\}, \boldsymbol{V}=\left\{\mathbf{u} \in \boldsymbol{H}_{0}^{1}(\Omega): \nabla_{\mathbf{x}} \cdot \mathbf{u}=0\right\}, \\
\boldsymbol{V}^{m}=\boldsymbol{V} \cap \boldsymbol{H}^{m}(\Omega),
\end{gathered}
$$

where $\mathscr{V}$ is dense in $\boldsymbol{H}, \boldsymbol{V}$, and $\boldsymbol{V}^{m}$. We also use the notation: $\mathbb{S}:=\mathbb{S}^{1}$, and let $\operatorname{Id} \in \mathbb{R}^{d \times d}$ denote the unit matrix. $A \hookrightarrow B$ (or $A \hookrightarrow \hookrightarrow B$ ) denotes that $A$ is continuously (or compactly) embedded in $B . f_{\tau} \rightarrow(\rightarrow$ or $\stackrel{*}{-}) f$ in $A$ denotes a sequence $\left\{f_{\tau}\right\}_{\tau>0} \subset A$ converges strongly (weakly or weakly star) to $f$ in $A$ as $\tau \rightarrow 0$. $C(a, b, \cdots)$ denotes a constant only dependent on $a, b, \ldots$.

To tackle the coupling term in (1.1), we need the following lemma of integrationby-parts.

Lemma 2.1 ([5], Lemma 1.1). Let $f \in W^{1,1}\left(\mathbb{S}^{d-1}\right)$ and $X \in \mathbb{R}^{d \times d}$ be a constant matrix with $\operatorname{tr}(X)=0$. Then

$$
\int_{\mathbb{S}^{d-1}}[(\mathrm{Id}-\mathbf{n} \otimes \mathbf{n}) X \mathbf{n}] \cdot \nabla_{\mathbf{n}} f d \mathbf{n}=\int_{\mathbb{S}^{d-1}}(d \mathbf{n} \otimes \mathbf{n}-\mathrm{Id}) f: X d \mathbf{n} .
$$

We let $\digamma(s):=s(\ln (s)-1)+1, s \in[0, \infty)$, and define some cut-off functions below. These cut-off functions will be used in the approximate problem, the entropy estimate, and the $L^{2}$-estimate in Section 3. 
Definition 2.2. Let $L>1$. Define

$$
\begin{aligned}
E^{L}(s): & = \begin{cases}0, & \text { if } s \leq 0, \\
s, & \text { if } 0 \leq s \leq L, \\
L, & \text { if } s \geq L ;\end{cases} \\
\digamma^{L}(s): & = \begin{cases}s(\ln (s)-1)+1, & 0 \leq s \leq L, \\
\frac{s^{2}-L^{2}}{2 L}+s(\ln (L)-1)+1, & s \geq L ;\end{cases} \\
G^{L}(s): & = \begin{cases}\frac{s^{2}}{2}, & s \leq L, \\
\frac{L^{2}}{2}+L(s-L), & s \geq L .\end{cases}
\end{aligned}
$$

With some elementary computations, one could verify the following properties (also see Barrett and Suli [2]-[3] for some of them).

Lemma 2.3. Let $L>1$. Then

$$
\begin{aligned}
& E^{L} \in C^{0,1}(\mathbb{R}) ; G^{L} \in C^{1,1}(\mathbb{R}) ; \digamma^{L} \in C^{2,1}\left(\mathbb{R}^{+}\right) \cap C([0, \infty)), \\
& \left(G^{L}\right)^{\prime}(s)=E^{L}(s), G^{L}(s) \leq \frac{s^{2}}{2}, \forall s \in[0, \infty), \\
& \digamma^{L}(s) \geq \digamma(s), \forall s \in[0, \infty), \\
& \left(\digamma^{L}\right)^{\prime \prime}(s)=\left[E^{L}(s)\right]^{-1} \geq s^{-1}, \forall s \in \mathbb{R}^{+}, \\
& \left(\digamma^{L}\right)^{\prime \prime}(s+\alpha) \leq \frac{1}{\alpha}, \forall \alpha \in(0,1), \forall s \in[0, \infty), \\
& \forall s \in[0, \infty), \lim _{L \rightarrow \infty} E^{L}(s)=s, \\
& \digamma^{L}\left(E^{L}(s)+\alpha\right) \leq \alpha+\frac{\alpha^{2}}{2}+\digamma(s+\alpha), \forall \alpha \in(0,1), \forall s \in[0, \infty) .
\end{aligned}
$$

The global weak solutions to the 2D Stokes-type model are defined as below.

Definition 2.4. Suppose $f_{0} \in L^{1}(\Omega \times \mathbb{S})$ and $f_{0} \geq 0$ a.e. on $\Omega \times \mathbb{S}$. A pair of measurable functions $(\mathbf{u}, f)$ is called a global weak solution to the $2 D$ Stokes-type model with boundary conditions (1.6) and initial condition (1.15) if

$$
\begin{aligned}
& \mathbf{u} \in L^{2}(0, \infty ; \boldsymbol{V}), f \geq 0 \text { a.e. on }[0, \infty) \times \Omega \times \mathbb{S}, \\
& f \in L^{\infty}\left(0, \infty ; L^{1}(\Omega \times \mathbb{S})\right), \sqrt{f} \in L^{2}\left(0, \infty ; H^{1}(\Omega \times \mathbb{S})\right),
\end{aligned}
$$

and for any $\mathbf{v} \in C_{0}^{\infty}((0, \infty) \times \Omega)$ with $\nabla_{\mathbf{x}} \cdot \mathbf{v}=0$,

$$
\begin{aligned}
& \int_{0}^{\infty} \int_{\Omega} \nabla_{\mathbf{x}} \mathbf{u}: \nabla_{\mathbf{x}} \mathbf{v} d \mathbf{x} d t \\
= & -\int_{0}^{\infty} \int_{\Omega \times \mathbb{S}}(2 \mathbf{n} \otimes \mathbf{n}-I d) f: \nabla_{\mathbf{x}} \mathbf{v} d \mathbf{n} d \mathbf{x} d t-\int_{0}^{\infty} \int_{\Omega \times \mathbb{S}} f \mathbf{e}_{2} \cdot \mathbf{v} d \mathbf{n} d \mathbf{x} d t ;
\end{aligned}
$$

also, for any $\varphi \in C_{0}^{\infty}([0, \infty) \times \bar{\Omega} \times \mathbb{S})$,

$$
\begin{aligned}
& -\int_{0}^{\infty} \int_{\Omega \times \mathbb{S}} f \partial_{t} \varphi d \mathbf{n} d \mathbf{x} d t-\int_{0}^{\infty} \int_{\Omega \times \mathbb{S}}(\mathbf{u} f) \cdot \nabla_{\mathbf{x}} \varphi d \mathbf{n} d \mathbf{x} d t+\int_{0}^{\infty} \int_{\Omega \times \mathbb{S}} \nabla_{\mathbf{n}} f \cdot \nabla_{\mathbf{n}} \varphi d \mathbf{n} d \mathbf{x} d t \\
= & \int_{0}^{\infty} \int_{\Omega \times \mathbb{S}}\left[(I d-\mathbf{n} \otimes \mathbf{n}) \nabla_{\mathbf{x}} \mathbf{u n} f\right] \cdot \nabla_{\mathbf{n}} \varphi d \mathbf{n} d \mathbf{x} d t
\end{aligned}
$$




$$
-\int_{0}^{\infty} \int_{\Omega \times \mathbb{S}}(I d+\mathbf{n} \otimes \mathbf{n})\left(\mathbf{e}_{2} f+\nabla_{\mathbf{x}} f\right) \cdot \nabla_{\mathbf{x}} \varphi d \mathbf{n} d \mathbf{x} d t+\int_{\Omega \times \mathbb{S}} f_{0}(\mathbf{x}, \mathbf{n}) \varphi(0, \mathbf{x}, \mathbf{n}) d \mathbf{n} d \mathbf{x} .
$$

REMARK 2.5. It follows from the Gagliardo-Nirenberg inequality that

$$
\|\sqrt{f}\|_{L^{4}\left(\Omega ; L^{2}(\mathbb{S})\right)} \leq C\|\sqrt{f}\|_{H^{1}\left(\Omega ; L^{2}(\mathbb{S})\right)}^{1 / 2}\|\sqrt{f}\|_{L^{2}\left(\Omega ; L^{2}(\mathbb{S})\right)}^{1 / 2},
$$

and hence by the Hölder inequality that

$$
\|\sqrt{f}\|_{L^{4}\left(0, \infty ; L^{4}\left(\Omega ; L^{2}(\mathbb{S})\right)\right)} \leq C\|\sqrt{f}\|_{L^{2}\left(0, \infty ; H^{1}(\Omega \times \mathbb{S})\right)}^{1 / 2}\|\sqrt{f}\|_{L^{\infty}\left(0, \infty ; L^{2}(\Omega \times \mathbb{S})\right)}^{1 / 2} .
$$

This and (2.9) yield $f \in L^{2}\left((0, \infty) \times \Omega ; L^{1}(\mathbb{S})\right)$. Therefore the $\nabla_{\mathbf{x}} \mathbf{u n} f$-related integral makes sense.

\section{Existence of a global weak solution}

In this section, we apply Barrett and Süli [2]-[3]'s cut-off techniques with some improvements and follow the usual procedure in proving the existence of global weak solutions.

First we use a semi-implicit scheme to construct a sequence of approximate solutions, where the Leray-Schauder fixed point theorem and cut-off techniques are used. In the construction of approximate solutions, our cut-off function is motivated by but different from that of Barrett and Süli [2]-[3]. First Barrett and Süli [2]-[3] used a cut-off only from above by $L>1$, then they used another cut-off from below by $\delta>0$. They established the uniform estimates for $\delta$ and took the limit $\delta \rightarrow 0$. It seems that their whole process is quite involved. However, we used a cut-off function by chopping off from above by $L>1$ and from below by 0 for the drag-term (see Definition 2.2). This single cut-off function is sufficient for the proof of existence for approximate solutions.

Then we use compactness to show that these constructed approximate solutions converge to a weak solution. In order to apply the time-space compactness theorems with assumptions on derivatives (such as Aubin-Lions-Simon lemma [24, Theorem 5] and the Dubinskii lemma [12, Theorem 1]), the traditional Rothe method for evolutionary PDEs (see [22] and [18]) is necessary and requires the construction of linear interpolation functions (also known as Rothe functions). However, the approach of the Rothe functions is fairly indirect and sometimes tedious, requiring more estimates and sometimes even more regularity assumptions on the initial data. In contrast, our approach is to apply Theorem 1 of Dreher-Jüngel [11], which consists of a nonlinear and a linear time-space compactness theorem with simple piecewise-constant functions of $t$, instead of the more complicated Rothe functions.

Now we state our main result.

TheOREm 3.1. Suppose $f_{0} \in L^{2}(\Omega \times \mathbb{S})$ and $f_{0} \geq 0$ a.e. on $\Omega \times \mathbb{S}$. Then the initialboundary problem of $2 D$ Stokes-type model has a global weak solution $(\mathbf{u}, f)$ which satisfies

$$
\begin{aligned}
& \mathbf{u} \in L_{l o c}^{\infty}(0, \infty ; \boldsymbol{V}) \cap L_{l o c}^{2}\left(0, \infty ; \boldsymbol{V}^{2}\right) \\
& f \in L_{l o c}^{\infty}\left(0, \infty ; L^{2}(\Omega \times \mathbb{S})\right) \cap L_{l o c}^{2}\left(0, \infty ; H^{1}(\Omega \times \mathbb{S})\right) \\
& f \in H_{l o c}^{1}\left(0, \infty ;\left(H^{1}(\Omega \times \mathbb{S})\right)^{\prime}\right)
\end{aligned}
$$


and for a.e. $t \in[0, \infty)$,

$$
\begin{aligned}
& \int_{\Omega \times \mathbb{S}} \digamma(f(t)) d \mathbf{n} d \mathbf{x}+\int_{0}^{t}\left\|\nabla_{\mathbf{x}} \mathbf{u}(s)\right\|_{L^{2}(\Omega)}^{2} d s \\
& \quad+2 \int_{0}^{t}\left(\left\|\nabla_{\mathbf{x}} \sqrt{f(s)}\right\|_{L^{2}(\Omega \times \mathbb{S})}^{2}+\left\|\nabla_{\mathbf{n}} \sqrt{f(s)}\right\|_{L^{2}(\Omega \times \mathbb{S})}^{2}\right) d s \\
& \leq \int_{\Omega \times \mathbb{S}} \digamma\left(f_{0}\right) d \mathbf{n} d \mathbf{x}+C\left\|f_{0}\right\|_{L^{1}(\Omega \times \mathbb{S})}^{2} .
\end{aligned}
$$

REMARK 3.2. Although the sedimentation problem for dilute rod-like particles we study here is in the Stokes regime, it is a mathematically interesting question to ask if the above result is still valid when the Stokes equation is replaced by the Navier-Stokes equation. There are some technical difficulties in solving this problem. We cannot directly obtain (3.20), and hence (3.22) in the approximate problem. Therefore we are not able to get (3.45), and prove the uniform $L^{2}$-estimate (3.38) by applying the discrete Gronwall inequality.

3.1. Approximate problem. In the construction of the approximate problem, a cut-off function chopping off above by some $L>1$ and chopping off below by 0 is used to ensure the boundedness of the linear functional (3.9) for the discrete Fokker-Planck equation required by the Lax-Milgram theorem, and the boundedness estimates for the existence of fixed-point solutions needed by the Leray-Schauder fixed point theorem. Using this effective cut-off, we obtain the existence of weak solutions in $\boldsymbol{V} \times H_{M}^{1}$ for the approximate problem, and then by applying the standard method for the resulting elliptic equation we get the nonnegativity of approximate distribution functions.

For any fixed $0<\tau<<1$ and for any $k \in \mathbb{N}$, given $f^{k-1}$, the approximate problem for the 2D Stokes-type model with cut-off reads

$$
\begin{aligned}
& \int_{\Omega} \nabla_{\mathbf{x}} \mathbf{u}^{k}: \nabla_{\mathbf{x}} \mathbf{v} d \mathbf{x}=-\int_{\Omega \times \mathbb{S}}(2 \mathbf{n} \otimes \mathbf{n}-\mathrm{Id}) f^{k}: \nabla_{\mathbf{x}} \mathbf{v} d \mathbf{n} d \mathbf{x}-\int_{\Omega \times \mathbb{S}} f^{k} \mathbf{e}_{2} \cdot \mathbf{v} d \mathbf{n} d \mathbf{x}, \forall \mathbf{v} \in \boldsymbol{V} \\
& \quad \int_{\Omega \times \mathbb{S}} \frac{f^{k}-f^{k-1}}{\tau} \varphi d \mathbf{n} d \mathbf{x}-\int_{\Omega \times \mathbb{S}}\left(\mathbf{u}^{k} f^{k}\right) \cdot \nabla_{\mathbf{x}} \varphi d \mathbf{n} d \mathbf{x}+\int_{\Omega \times \mathbb{S}} \nabla_{\mathbf{n}} f^{k} \cdot \nabla_{\mathbf{n}} \varphi d \mathbf{n} d \mathbf{x} \\
& =\int_{\Omega \times \mathbb{S}}\left[(\mathrm{Id}-\mathbf{n} \otimes \mathbf{n}) \nabla_{\mathbf{x}} \mathbf{u}^{k} \mathbf{n}\right] E^{\tau^{-\frac{1}{4}}}\left(f^{k}\right) \cdot \nabla_{\mathbf{n}} \varphi d \mathbf{n} d \mathbf{x} \\
& \quad-\int_{\Omega \times \mathbb{S}}(\mathrm{Id}+\mathbf{n} \otimes \mathbf{n})\left[\mathbf{e}_{2} E^{\tau^{-\frac{1}{4}}}\left(f^{k}\right)+\nabla_{\mathbf{x}} f^{k}\right] \cdot \nabla_{\mathbf{x}} \varphi d \mathbf{n} d \mathbf{x}, \forall \varphi \in H^{1}(\Omega \times \mathbb{S})
\end{aligned}
$$

in which $E^{\tau^{-\frac{1}{4}}}$ is the cut-off function given by Definition 2.2.

DEFINITION 3.3.

$$
Z:=\left\{f \in L^{2}(\Omega \times \mathbb{S}): f \geq 0 \text { a.e.on } \Omega \times \mathbb{S}\right\} .
$$

Proposition 3.4. Let $f^{k-1} \in Z$. Then there exists $\left(\mathbf{u}^{k}, f^{k}\right) \in \boldsymbol{V} \times\left(Z \cap H^{1}(\Omega \times \mathbb{S})\right)$ which solves (3.5)-(3.6). 
Proof. Step 1. Let $\bar{f} \in L^{2}(\Omega \times \mathbb{S})$. We claim that there exists a unique element $\mathbf{u} \in \boldsymbol{V}$ such that

$$
a(\mathbf{u}, \mathbf{v})=A(\bar{f})(\mathbf{v}), \forall \mathbf{v} \in \boldsymbol{V},
$$

where $a(\mathbf{u}, \mathbf{v})=\int_{\Omega} \nabla_{\mathbf{x}} \mathbf{u}: \nabla_{\mathbf{x}} \mathbf{v} d \mathbf{x}, \forall \mathbf{u}, \mathbf{v} \in \boldsymbol{V}$, and

$$
A(\bar{f})(\mathbf{v})=-\int_{\Omega \times \mathbb{S}}(2 \mathbf{n} \otimes \mathbf{n}-\mathrm{Id}) \bar{f}: \nabla_{\mathbf{x}} \mathbf{v} d \mathbf{n} d \mathbf{x}-\int_{\Omega \times \mathbb{S}} \bar{f} \mathbf{e}_{2} \cdot \mathbf{v} d \mathbf{n} d \mathbf{x}, \forall \mathbf{v} \in \boldsymbol{V} .
$$

Then thanks to the Poincaré inequality and $\left\|\int_{\mathbb{S}}(2 \mathbf{n} \otimes \mathbf{n}-\mathrm{Id}) \bar{f} d \mathbf{n}\right\|_{L^{2}(\Omega)} \leq$ $C\|\bar{f}\|_{L^{2}(\Omega \times \mathbb{S})}$, we have that $a(\cdot, \cdot)$ is a bounded, coercive bilinear functional on $\boldsymbol{V}$ and $A(\bar{f}) \in \boldsymbol{V}^{\prime}$. Hence by the Lax-Milgram theorem, we finish the proof of Step 1 .

Step 2. We prove that for such $\bar{f} \in L^{2}(\Omega \times \mathbb{S})$ and solutions $\mathbf{u} \in \boldsymbol{V}$ in (3.8), there exists a unique element $f \in H^{1}(\Omega \times \mathbb{S})$ such that

$$
b(\mathbf{u})(f, \varphi)=B(\bar{f}, \mathbf{u})(\varphi), \forall \varphi \in H^{1}(\Omega \times \mathbb{S}),
$$

where

$$
\begin{aligned}
& b(\mathbf{u})(f, \varphi)= \int_{\Omega \times \mathbb{S}} f \varphi d \mathbf{n} d \mathbf{x}+\tau \int_{\Omega \times \mathbb{S}}\left[(\operatorname{Id}+\mathbf{n} \otimes \mathbf{n}) \nabla_{\mathbf{x}} f \cdot \nabla_{\mathbf{x}} \varphi+\nabla_{\mathbf{n}} f \cdot \nabla_{\mathbf{n}} \varphi\right] d \mathbf{n} d \mathbf{x} \\
&-\tau \int_{\Omega \times \mathbb{S}}(\mathbf{u} f) \cdot \nabla_{\mathbf{x}} \varphi d \mathbf{n} d \mathbf{x}, \forall f, \varphi \in H^{1}(\Omega \times \mathbb{S}) ; \\
& B(\bar{f}, \mathbf{u})(\varphi)=\int_{\Omega \times \mathbb{S}} f^{k-1} \varphi d \mathbf{n} d \mathbf{x}-\tau \int_{\Omega \times \mathbb{S}}\left[(\operatorname{Id}+\mathbf{n} \otimes \mathbf{n}) \mathbf{e}_{2}\right] E^{\tau^{-\frac{1}{4}}}(\bar{f}) \cdot \nabla_{\mathbf{x}} \varphi d \mathbf{n} d \mathbf{x} \\
& \quad+\tau \int_{\Omega \times \mathbb{S}}\left[(\operatorname{Id}-\mathbf{n} \otimes \mathbf{n}) \nabla_{\mathbf{x}} \mathbf{u n}\right] E^{\tau^{-\frac{1}{4}}}(\bar{f}) \cdot \nabla_{\mathbf{n}} \varphi d \mathbf{n} d \mathbf{x}, \forall \varphi \in H^{1}(\Omega \times \mathbb{S}) .
\end{aligned}
$$

Indeed, by noting $H^{1}(\Omega) \hookrightarrow L^{6}(\Omega), H^{1}(\Omega \times \mathbb{S}) \hookrightarrow L^{3}(\Omega \times \mathbb{S})$, and $\nabla_{\mathbf{x}} \cdot \mathbf{u}=0$, we have

$$
\begin{aligned}
\left|\int_{\Omega \times \mathbb{S}}(\mathbf{u} f) \cdot \nabla_{\mathbf{x}} \varphi d \mathbf{n} d \mathbf{x}\right| & \leq\|\mathbf{u}\|_{L^{6}(\Omega)}\|f\|_{L^{3}(\Omega \times \mathbb{S})}\left\|\nabla_{\mathbf{x}} \varphi\right\|_{L^{2}(\Omega \times \mathbb{S})} \\
& \leq C\|f\|_{H^{1}(\Omega \times \mathbb{S})}\|\varphi\|_{H^{1}(\Omega \times \mathbb{S})}
\end{aligned}
$$

and

$$
\int_{\Omega \times \mathbb{S}}(\mathbf{u} f) \cdot \nabla_{\mathbf{x}} f d \mathbf{n} d \mathbf{x}=0
$$

Because $\operatorname{Id}+\mathbf{n} \otimes \mathbf{n}$ is a positive definite matrix with smallest eigenvalue 1 , we have

$$
\left|\nabla_{\mathbf{x}} f\right|^{2} \leq(\mathrm{Id}+\mathbf{n} \otimes \mathbf{n}) \nabla_{\mathbf{x}} f \cdot \nabla_{\mathbf{x}} f .
$$

Therefore $b(\mathbf{u})(\cdot, \cdot)$ is a bounded and coercive bilinear functional on $H^{1}(\Omega \times \mathbb{S})$. It follows from $0 \leq E^{\tau^{-\frac{1}{4}}}(s) \leq \tau^{-\frac{1}{4}}(\forall s \in \mathbb{R})$ that $B(\bar{f}, \mathbf{u}) \in\left(H^{1}(\Omega \times \mathbb{S})\right)^{\prime}$. We thus finish the proof of Step 2 by the Lax-Milgram theorem.

Step 3. Define the mapping $\Phi: L^{2}(\Omega \times \mathbb{S}) \rightarrow L^{2}(\Omega \times \mathbb{S})$ by $\Phi(\bar{f})=f \in H^{1}(\Omega \times \mathbb{S})$ via the procedure (3.8) and (3.9). By the Leray-Schauder fixed-point theorem (see [14], Theorem 11.3), we obtain a solution $f$ to $\Phi(f)=f$, and hence a fixed-point 
solution $(\mathbf{u}, f) \in V \times H^{1}(\Omega \times \mathbb{S})$ to (3.5) and (3.6). To prove this, we only need to show the following three claims.

Claim 1. $\Phi: L^{2}(\Omega \times \mathbb{S}) \rightarrow L^{2}(\Omega \times \mathbb{S})$ is continuous.

Claim 2. $\Phi$ is compact. $\mathbb{S})$.

Claim 3. $\Lambda:=\left\{f \in L^{2}(\Omega \times \mathbb{S}): f=\sigma \Phi(f)\right.$ for some $\left.\sigma \in(0,1]\right\}$ is bounded in $L^{2}(\Omega \times$

Proof. [Proof of Claim 1.] Set $f:=\Phi(\bar{f})$ and $f_{i}:=\Phi\left(\bar{f}_{i}\right), i \in \mathbb{N}$. If

$$
\bar{f}_{i} \rightarrow \bar{f} \text { in } L^{2}(\Omega \times \mathbb{S}) \text {, as } i \rightarrow \infty,
$$

we need to show

$$
f_{i} \rightarrow f \text { in } L^{2}(\Omega \times \mathbb{S}) \text {, as } i \rightarrow \infty .
$$

Indeed, for $\bar{f}$ and $\bar{f}_{i}$, in view of the definition of $\Phi$, there exist unique $\mathbf{u} \in \boldsymbol{V}$ and $\mathbf{u}_{i} \in \boldsymbol{V}$ such that

$$
\begin{array}{lll}
a(\mathbf{u}, \mathbf{v})=A(\bar{f})(\mathbf{v}), & a\left(\mathbf{u}_{i}, \mathbf{v}\right)=A\left(\bar{f}_{i}\right)(\mathbf{v}), & \forall \mathbf{v} \in \boldsymbol{V}, \\
b(\mathbf{u})(f, \varphi)=B(\bar{f}, \mathbf{u})(\varphi), & b\left(\mathbf{u}_{i}\right)\left(f_{i}, \varphi\right)=B\left(\bar{f}_{i}, \mathbf{u}_{i}\right)(\varphi), & \forall \varphi \in H^{1}(\Omega \times \mathbb{S}) .
\end{array}
$$

By subtracting the terms in (3.14), we obtain

$$
a\left(\mathbf{u}_{i}, \mathbf{v}\right)-a(\mathbf{u}, \mathbf{v})=A\left(\bar{f}_{i}\right)(\mathbf{v})-A(\bar{f})(\mathbf{v}),
$$

and by taking $\mathbf{v}=\mathbf{u}_{i}-\mathbf{u}$, we have that

$$
\int_{\Omega}\left|\nabla_{\mathbf{x}} \mathbf{u}_{i}-\nabla_{\mathbf{x}} \mathbf{u}\right|^{2} d \mathbf{x} \leq C \int_{\Omega \times \mathbb{S}}\left|\bar{f}_{i}-\bar{f}\right|\left(\left|\nabla_{\mathbf{x}} \mathbf{u}_{i}-\nabla_{\mathbf{x}} \mathbf{u}\right|+\left|\mathbf{u}_{i}-\mathbf{u}\right|\right) d \mathbf{n} d \mathbf{x} .
$$

Then from the Cauchy-Schwartz inequality one has that $\left\|\mathbf{u}_{i}-\mathbf{u}\right\|_{H^{1}(\Omega)}^{2} \leq C \| \bar{f}_{i}-$ $\bar{f} \|_{L^{2}(\Omega \times \mathbb{S})}^{2}$. Thus (3.12) yields

$$
\mathbf{u}_{i} \rightarrow \mathbf{u} \text { in } \boldsymbol{H}^{1}(\Omega) \text {, as } i \rightarrow \infty .
$$

By (3.15), using the same procedure as above and noting that

$$
\begin{aligned}
& \int_{\Omega \times \mathbb{S}}\left(\mathbf{u}_{i} f_{i}-\mathbf{u} f\right) \cdot \nabla_{\mathbf{x}}\left(f_{i}-f\right) d \mathbf{n} d \mathbf{x} \\
= & \int_{\Omega \times \mathbb{S}} \mathbf{u}_{i}\left(f_{i}-f\right) \cdot \nabla_{\mathbf{x}}\left(f_{i}-f\right) d \mathbf{n} d \mathbf{x}+\int_{\Omega \times \mathbb{S}}\left(\mathbf{u}_{i}-\mathbf{u}\right) f \cdot \nabla_{\mathbf{x}}\left(f_{i}-f\right) d \mathbf{n} d \mathbf{x} \\
= & \int_{\Omega \times \mathbb{S}}\left(\mathbf{u}_{i}-\mathbf{u}\right) f \cdot \nabla_{\mathbf{x}}\left(f_{i}-f\right) d \mathbf{n} d \mathbf{x},
\end{aligned}
$$

one has

$$
\begin{aligned}
& \quad\left\|f_{i}-f\right\|_{H^{1}(\Omega \times \mathbb{S})}^{2} \\
& \leq C\left(\left\|E^{L}\left(\bar{f}_{i}\right)-E^{L}(\bar{f})\right\|_{L^{2}(\Omega \times \mathbb{S})}^{2}+\left\|\left(\mathbf{u}_{i}-\mathbf{u}\right) f\right\|_{L^{2}(\Omega \times \mathbb{S})}^{2}\right. \\
& \left.\quad+\left\|\left[(\operatorname{Id}-\mathbf{n} \otimes \mathbf{n}) \nabla_{\mathbf{x}} \mathbf{u}_{i} \mathbf{n}\right] E^{L}\left(\bar{f}_{i}\right)-\left[(\operatorname{Id}-\mathbf{n} \otimes \mathbf{n}) \nabla_{\mathbf{x}} \mathbf{u n}\right] E^{L}(\bar{f})\right\|_{L^{2}(\Omega \times \mathbb{S})}^{2}\right)
\end{aligned}
$$




$$
=: C\left(I_{1}^{2}+I_{2}^{2}+I_{3}^{2}\right) .
$$

We have from $E^{\tau^{-\frac{1}{4}}} \in C^{0,1}(\mathbb{R})$ with Lipschitz coefficient 1 that $I_{1} \leq\left\|\bar{f}_{i}-\bar{f}\right\|_{L^{2}(\Omega \times \mathbb{S})}$. It follows from (3.16) that

$$
\begin{aligned}
I_{2} \leq C\left\|\mathbf{u}_{i}-\mathbf{u}\right\|_{L^{6}(\Omega)}\|f\|_{L^{3}(\Omega \times \mathbb{S})} & \leq C\left\|\mathbf{u}_{i}-\mathbf{u}\right\|_{H^{1}(\Omega)}\|f\|_{H^{1}(\Omega \times \mathbb{S})} \\
& \leq C\left\|\mathbf{u}_{i}-\mathbf{u}\right\|_{H^{1}(\Omega)} \rightarrow 0 \text { as } i \rightarrow 0 .
\end{aligned}
$$

Now we estimate $I_{3}$.

$$
\begin{aligned}
I_{3} \leq & \left\|\left[(\mathrm{Id}-\mathbf{n} \otimes \mathbf{n})\left(\nabla_{\mathbf{x}} \mathbf{u}_{i}-\nabla_{\mathbf{x}} \mathbf{u}\right) \mathbf{n}\right] E^{\tau^{-\frac{1}{4}}}\left(\bar{f}_{i}\right)\right\|_{L^{2}(\Omega \times \mathbb{S})} \\
& \quad+\left\|\left[(\operatorname{Id}-\mathbf{n} \otimes \mathbf{n}) \nabla_{\mathbf{x}} \mathbf{u n}\right]\left[E^{\tau^{-\frac{1}{4}}}\left(\bar{f}_{i}\right)-E^{\tau^{-\frac{1}{4}}}(\bar{f})\right]\right\|_{L^{2}(\Omega \times \mathbb{S})} \\
= & : I_{3,1}+I_{3,2} .
\end{aligned}
$$

However (3.16) yields $I_{3,1} \leq C \tau^{-\frac{1}{4}}\left\|\nabla_{\mathbf{x}}\left(\mathbf{u}_{i}-\mathbf{u}\right)\right\|_{L^{2}(\Omega)} \rightarrow 0$ as $i \rightarrow \infty$. We only need to deal with $I_{3,2}$. In fact, because $(\operatorname{Id}-\mathbf{n} \otimes \mathbf{n}) \nabla_{\mathbf{x}} \mathbf{u n} \in \boldsymbol{L}^{2}(\Omega)$ and $\boldsymbol{C}^{\infty}(\Omega)$ is dense in $\boldsymbol{L}^{2}(\Omega)$, we have that

$$
\forall \varepsilon>0, \exists \mathbf{w} \in \boldsymbol{C}^{\infty}(\Omega) \text { such that }\left\|(\mathrm{Id}-\mathbf{n} \otimes \mathbf{n}) \nabla_{\mathbf{x}} \mathbf{u n}-\mathbf{w}\right\|_{L^{2}(\Omega)}<\varepsilon .
$$

Moreover, using (3.12) and the fact that $E^{\tau^{-\frac{1}{4}}} \in C^{0,1}(\mathbb{R})$ with Lipschitz coefficient 1,

$$
\exists i_{0} \in \mathbb{N}, \forall i>i_{0},\left\|\mathbf{w}\left[E^{\tau^{-\frac{1}{4}}}\left(\bar{f}_{i}\right)-E^{\tau^{-\frac{1}{4}}}(\bar{f})\right]\right\|_{L^{2}(\Omega \times \mathbb{S})} \leq\|\mathbf{w}\|_{L^{\infty}(\Omega)}\left\|\bar{f}_{i}-\bar{f}\right\|_{L^{2}(\Omega \times \mathbb{S})}<\varepsilon .
$$

Therefore

$$
\begin{aligned}
I_{3} \leq \| & {\left[(\operatorname{Id}-\mathbf{n} \otimes \mathbf{n}) \nabla_{\mathbf{x}} \mathbf{u n}-\mathbf{w}\right]\left[E^{\tau^{-\frac{1}{4}}}\left(\bar{f}_{i}\right)-E^{\tau^{-\frac{1}{4}}}(\bar{f})\right] \|_{L^{2}(\Omega \times \mathbb{S})} } \\
& +\left\|\mathbf{w}\left[E^{\tau^{-\frac{1}{4}}}\left(\bar{f}_{i}\right)-E^{\tau^{-\frac{1}{4}}}(\bar{f})\right]\right\|_{L^{2}(\Omega \times \mathbb{S})} \\
& \leq \tau^{-\frac{1}{4}}\left\|(\operatorname{Id}-\mathbf{n} \otimes \mathbf{n}) \nabla_{\mathbf{x}} \mathbf{u n}-\mathbf{w}\right\|_{L^{2}(\Omega \times \mathbb{S})}+\left\|\mathbf{w}\left[E^{\tau^{-\frac{1}{4}}}\left(\bar{f}_{i}\right)-E^{\tau^{-\frac{1}{4}}}(\bar{f})\right]\right\|_{L^{2}(\Omega \times \mathbb{S})}<C(\tau) \varepsilon .
\end{aligned}
$$

Consequently $f_{i} \rightarrow f$ in $H^{1}(\Omega \times \mathbb{S})$ and hence (3.13) holds. This ends the proof of Claim 1.

Proof. [Proof of Claim 2.] It is quite easy to deduce that

$$
\exists C(\tau)>0, \forall \bar{f} \in L^{2}(\Omega \times \mathbb{S}), \quad\|\Phi(\bar{f})\|_{H^{1}(\Omega \times \mathbb{S})} \leq C(\tau)\left(1+\|\bar{f}\|_{L^{2}(\Omega \times \mathbb{S})}\right) .
$$

Thus we have from $H^{1}(\Omega \times \mathbb{S}) \hookrightarrow \hookrightarrow L^{2}(\Omega \times \mathbb{S})$ that Claim 2 holds.

Proof. [Proof of Claim 3.] For any $f \in \Lambda$, there exists a unique $\mathbf{u} \in \boldsymbol{V}$ such that

$$
\begin{aligned}
& a(\mathbf{u}, \mathbf{v})=A(f)(v), \forall \mathbf{v} \in \boldsymbol{V}, \\
& b(\mathbf{u})(f, \varphi)=\sigma B(f, \mathbf{u})(\varphi), \forall \varphi \in H^{1}(\Omega \times \mathbb{S}) .
\end{aligned}
$$

Taking $\mathbf{v}=\mathbf{u}$ in (3.17), we have from the Cauchy-Schwartz inequality and the Poincaré inequality that

$$
\int_{\Omega}\left|\nabla_{\mathbf{x}} \mathbf{u}\right|^{2} d \mathbf{x} \leq C \int_{\Omega \times \mathbb{S}}|f|\left(\left|\nabla_{\mathbf{x}} \mathbf{u}\right|+|\mathbf{u}|\right) d \mathbf{n} d \mathbf{x} \leq \frac{1}{2} \int_{\Omega}\left|\nabla_{\mathbf{x}} \mathbf{u}\right|^{2} d \mathbf{x}+C\|f\|_{L^{2}\left(\Omega ; L^{1}(\mathbb{S})\right)}^{2} .
$$


Therefore

$$
\int_{\Omega}\left|\nabla_{\mathbf{x}} \mathbf{u}\right|^{2} d \mathbf{x} \leq C\|f\|_{L^{2}(\Omega \times \mathbb{S})}^{2}
$$

Taking $\varphi=f$ in (3.18), we deduce from (3.10), (3.11), the Cauchy-Schwartz inequality, and the identity

$$
2(a-b) a=a^{2}+(a-b)^{2}-b^{2}, \forall a, b \in \mathbb{R},
$$

that

$$
\begin{aligned}
& \quad \frac{1}{2} \int_{\Omega \times \mathbb{S}}|f|^{2} d \mathbf{n} d \mathbf{x}+\tau \int_{\Omega \times \mathbb{S}}\left[\left|\nabla_{\mathbf{x}} f\right|^{2}+\left|\nabla_{\mathbf{n}} f\right|^{2}\right] d \mathbf{n} d \mathbf{x} \\
& \leq \frac{1}{2} \int_{\Omega \times \mathbb{S}}\left|\sigma f^{k-1}\right|^{2} d \mathbf{n} d \mathbf{x}-\sigma \tau \int_{\Omega \times \mathbb{S}}\left[(\mathrm{Id}+\mathbf{n} \otimes \mathbf{n}) \mathbf{e}_{2}\right] E^{\tau^{-\frac{1}{4}}}(f) \cdot \nabla_{\mathbf{x}} f d \mathbf{n} d \mathbf{x} \\
& \quad+\sigma \tau \int_{\Omega \times \mathbb{S}}\left[(\mathrm{Id}-\mathbf{n} \otimes \mathbf{n}) \nabla_{\mathbf{x}} \mathbf{u n}\right] E^{\tau^{-\frac{1}{4}}}(f) \cdot \nabla_{\mathbf{n}} f d \mathbf{n} d \mathbf{x} \\
& \leq \frac{1}{2} \int_{\Omega \times \mathbb{S}}\left|f^{k-1}\right|^{2} d \mathbf{n} d \mathbf{x}+C \tau \int_{\Omega \times \mathbb{S}}|f|\left|\nabla_{\mathbf{x}} f\right| d \mathbf{n} d \mathbf{x}+C \tau^{\frac{3}{4}} \int_{\Omega \times \mathbb{S}}\left|\nabla_{\mathbf{x}} \mathbf{u}\right|\left|\nabla_{\mathbf{n}} f\right| d \mathbf{n} d \mathbf{x} \\
& \leq \frac{1}{2} \int_{\Omega \times \mathbb{S}}\left|f^{k-1}\right|^{2} d \mathbf{n} d \mathbf{x}+\frac{\tau}{2} \int_{\Omega \times \mathbb{S}}\left(\left|\nabla_{\mathbf{x}} f\right|^{2}+\left|\nabla_{\mathbf{n}} f\right|^{2}\right) d \mathbf{n} d \mathbf{x} \\
& \quad+C \tau \int_{\Omega \times \mathbb{S}}|f|^{2} d \mathbf{n} d \mathbf{x}+C \tau^{\frac{1}{2}} \int_{\Omega \times \mathbb{S}}\left|\nabla_{\mathbf{x}} \mathbf{u}\right|^{2} d \mathbf{n} d \mathbf{x} .
\end{aligned}
$$

Thus (3.20) and (3.21) yield

$$
\|f\|_{L^{2}(\Omega \times \mathbb{S})}^{2} \leq\left\|f^{k-1}\right\|_{L^{2}(\Omega \times \mathbb{S})}^{2}+C \tau^{\frac{1}{2}}\|f\|_{L^{2}(\Omega \times \mathbb{S})}^{2} .
$$

Noting that $C \tau^{\frac{1}{2}}<\frac{1}{2}$, we have

$$
\|f\|_{L^{2}(\Omega \times \mathbb{S})}^{2} \leq 2\left\|f^{k-1}\right\|_{L^{2}(\Omega \times \mathbb{S})}^{2}
$$

proving Claim 3.

Step 4. We prove the nonnegativity for $f$. For explicitness, we relabel $(\mathbf{u}, f)$ as $\left(\mathbf{u}^{k}, f^{k}\right)$.

In fact, set $\left[f^{k}\right]^{-}:=\min \left\{f^{k}, 0\right\}$. Then $\left[f^{k}\right]^{-} \in H^{1}(\Omega \times \mathbb{S})$. Choosing $\varphi=\left[f^{k}\right]^{-}$ in (3.6) and noting that $E^{\tau^{-\frac{1}{4}}}\left(f^{k}\right) \nabla_{\mathbf{x}}\left[f^{k}\right]^{-}=E^{\tau^{-\frac{1}{4}}}\left(f^{k}\right) \nabla_{\mathbf{n}}\left[f^{k}\right]^{-}=0$, we deduce from $\left|\nabla_{\mathbf{x}}\left[f^{k}\right]^{-}\right|^{2} \leq(\mathrm{Id}+\mathbf{n} \otimes \mathbf{n}) \nabla_{\mathbf{x}}\left[f^{k}\right]^{-} \cdot \nabla_{\mathbf{x}}\left[f^{k}\right]^{-}$that

$$
\begin{aligned}
& \int_{\Omega \times \mathbb{S}}\left|\left[f^{k}\right]^{-}\right|^{2} d \mathbf{n} d \mathbf{x}+\tau \int_{\Omega \times \mathbb{S}}\left[\left|\nabla_{\mathbf{x}}\left[f^{k}\right]^{-}\right|^{2}+\left|\nabla_{\mathbf{n}}\left[f^{k}\right]^{-}\right|^{2}\right] d \mathbf{n} d \mathbf{x} \\
\leq & \int_{\Omega \times \mathbb{S}} f^{k-1}\left[f^{k}\right]^{-} d \mathbf{n} d \mathbf{x} \leq 0 .
\end{aligned}
$$

Therefore $\left[f^{k}\right]^{-}=0$ a.e. on $\Omega \times \mathbb{S}$ and hence $f^{k} \geq 0$ a.e. on $\Omega \times \mathbb{S}$. Thus $f^{k} \in Z$. This finishes the proof of Proposition3.4. 
3.2. Entropy estimate, uniform in $\tau$ and time $t$. Suppose $f_{0} \in L^{2}(\Omega \times$ $\mathbb{S})$ ) and $f_{0} \geq 0$ a.e. on $\Omega \times \mathbb{S}$. Let $f^{0}=E^{\tau^{-\frac{1}{4}}}\left(f_{0}\right)$. Then $f^{0} \in Z$. Using Proposition 3.4 iteratively, we obtain a sequence of approximate solutions

$$
\left(\mathbf{u}^{k}, f^{k}\right) \in \boldsymbol{V} \times\left(Z \cap H^{1}(\Omega \times \mathbb{S})\right), \quad(k \in \mathbb{N})
$$

to (3.5)-(3.6). Choosing $\varphi=1$ in (3.6), one has the following lemma directly.

LEMMA 3.5.

$$
\sup _{k \in \mathbb{N}}\left\|f^{k}\right\|_{L^{1}(\Omega \times \mathbb{S})} \leq\left\|f_{0}\right\|_{L^{1}(\Omega \times \mathbb{S})} .
$$

We use $\left(\digamma^{\tau^{-\frac{1}{4}}}\right)^{\prime}\left(f^{k}+\alpha\right)$ as test function and then let $\alpha \rightarrow 0$, to deal with the singularity when $f_{k}(x, \mathbf{n})=0$ on some subset of $\Omega \times \mathbb{S}$. Another problem is tackling the term $\int_{\Omega \times \mathbb{S}}\left[(\operatorname{Id}-\mathbf{n} \otimes \mathbf{n}) \nabla_{\mathbf{x}} \mathbf{u}^{k} \mathbf{n}\right] \cdot \nabla_{\mathbf{n}} f^{k} d \mathbf{n} d \mathbf{x}$ in the proof. We apply Lemma 2.1 to solve this problem.

Lemma 3.6. For any $k \in \mathbb{N}$,

$$
\begin{aligned}
& \int_{\Omega \times \mathbb{S}} \digamma\left(f^{k}\right) d \mathbf{n} d \mathbf{x}+\tau \sum_{i=1}^{k}\left\|\nabla_{\mathbf{x}} \mathbf{u}^{i}\right\|_{L^{2}(\Omega)}^{2} \\
& \quad+2 \tau \sum_{i=1}^{k}\left(\left\|\nabla_{\mathbf{x}} \sqrt{f^{i}}\right\|_{L^{2}(\Omega \times \mathbb{S})}^{2}+\left\|\nabla_{\mathbf{n}} \sqrt{f^{i}}\right\|_{L^{2}(\Omega \times \mathbb{S})}^{2}\right) \\
& \leq \int_{\Omega \times \mathbb{S}} \digamma\left(f_{0}\right) d \mathbf{n} d \mathbf{x}+C\left\|f_{0}\right\|_{L^{1}(\Omega \times \mathbb{S})} .
\end{aligned}
$$

Proof. Let $\alpha \in(0,1)$ and denote $L:=\tau^{-\frac{1}{4}}$. Taking $\varphi=\left(\digamma^{L}\right)^{\prime}\left(f^{k}+\alpha\right)+\mathbf{x} \cdot \mathbf{e}_{2} \in$ $H^{1}(\Omega \times \mathbb{S})$ in (3.6), we have from the convexity of $\digamma^{L}$ that

$$
\begin{aligned}
& \int_{\Omega \times \mathbb{S}}\left[\digamma^{L}\left(f^{k}+\alpha\right)-\digamma^{L}\left(f^{k-1}+\alpha\right)\right] d \mathbf{n} d \mathbf{x}+\int_{\Omega \times \mathbb{S}}\left[f^{k} \mathbf{x} \cdot \mathbf{e}_{2}-f^{k-1} \mathbf{x} \cdot \mathbf{e}_{2}\right] d \mathbf{n} d \mathbf{x} \\
& \quad+\tau \int_{\Omega \times \mathbb{S}}\left|\nabla_{\mathbf{n}} f^{k}\right|^{2}\left(\digamma^{L}\right)^{\prime \prime}\left(f^{k}+\alpha\right) d \mathbf{n} d \mathbf{x} \\
& \leq \tau \int_{\Omega \times \mathbb{S}}\left(\mathbf{u}^{k} f^{k}\right) \cdot \nabla_{\mathbf{x}}\left[\left(\digamma^{L}\right)^{\prime}\left(f^{k}+\alpha\right)\right] d \mathbf{n} d \mathbf{x}+\tau \int_{\Omega \times \mathbb{S}}\left(\mathbf{u}^{k} f^{k}\right) \cdot \mathbf{e}_{2} d \mathbf{n} d \mathbf{x} \\
& \left.\quad+\tau \int_{\Omega \times \mathbb{S}}\left[E^{L}\left(f^{k}\right)\left(\digamma^{L}\right)^{\prime \prime}\left(f^{k}+\alpha\right)\right]\left[(\mathrm{Id}-\mathbf{n} \otimes \mathbf{n}) \nabla_{\mathbf{x}} \mathbf{u}^{k}\right) \mathbf{n}\right] \cdot \nabla_{\mathbf{n}} f^{k} d \mathbf{n} d \mathbf{x} \\
& \quad-\tau \int_{\Omega \times \mathbb{S}}(\mathrm{Id}+\mathbf{n} \otimes \mathbf{n})\left[\mathbf{e}_{2} E^{L}\left(f^{k}\right)+\nabla_{\mathbf{x}} f^{k}\right] \cdot\left[\mathbf{e}_{2}+\left(\digamma^{L}\right)^{\prime \prime}\left(f^{k}+\alpha\right) \nabla_{\mathbf{x}} f^{k}\right] d \mathbf{n} d \mathbf{x} \\
& =: J_{1}+J_{2}+J_{3}+J_{4} .
\end{aligned}
$$

Integrating by parts, one has from $\mathbf{u}^{k} \in \boldsymbol{V}$ that

$$
J_{1}=-\tau \int_{\Omega \times \mathbb{S}}\left(\mathbf{u}^{k} \cdot \nabla_{\mathbf{x}} f^{k}\right)\left(\digamma^{L}\right)^{\prime}\left(f^{k}+\alpha\right) d \mathbf{n} d \mathbf{x}=-\tau \int_{\Omega \times \mathbb{S}} \mathbf{u}^{k} \cdot \nabla_{\mathbf{x}}\left[\digamma^{L}\left(f^{k}+\alpha\right)\right] d \mathbf{n} d \mathbf{x}=0 .
$$

For $J_{3}$, one divides it into two parts as below:

$$
J_{3}=\tau \int_{\Omega \times \mathbb{S}}\left[E^{L}\left(f^{k}\right)\left(\digamma^{L}\right)^{\prime \prime}\left(f^{k}+\alpha\right)-1\right]\left[(\operatorname{Id}-\mathbf{n} \otimes \mathbf{n}) \nabla_{\mathbf{x}} \mathbf{u}^{k} \mathbf{n}\right] \cdot \nabla_{\mathbf{n}} f^{k} d \mathbf{n} d \mathbf{x}
$$




$$
\begin{aligned}
& \quad+\tau \int_{\Omega \times \mathbb{S}}\left[(\mathrm{Id}-\mathbf{n} \otimes \mathbf{n}) \nabla_{\mathbf{x}} \mathbf{u}^{k} \mathbf{n}\right] \cdot \nabla_{\mathbf{n}} f^{k} d \mathbf{n} d \mathbf{x} \\
= & : J_{3,1}+J_{3,2} .
\end{aligned}
$$

The Cauchy-Schwartz inequality, $E^{L} \in C^{0,1}(\mathbb{R})$ with Lipschitz coefficient $1,(2.4)$, and (2.5) imply

$$
\begin{aligned}
J_{3,1} & \leq C \tau \int_{\Omega \times \mathbb{S}}\left|\nabla_{\mathbf{x}} \mathbf{u}^{k}\right|\left|\nabla_{\mathbf{n}} f^{k}\right|\left|\left(\digamma^{L}\right)^{\prime \prime}\left(f^{k}+\alpha\right)\right|\left|E^{L}\left(f^{k}+\alpha\right)-E^{L}\left(f^{k}\right)\right| d \mathbf{n} d \mathbf{x} \\
& \leq C \sqrt{\alpha} \tau \int_{\Omega \times \mathbb{S}}\left|\nabla_{\mathbf{x}} \mathbf{u}^{k}\right|\left|\nabla_{\mathbf{n}} f^{k}\right| \sqrt{\left(\digamma^{L}\right)^{\prime \prime}\left(f^{k}+\alpha\right)} d \mathbf{n} d \mathbf{x} \\
& \leq C \alpha \tau \int_{\Omega}\left|\nabla_{\mathbf{x}} \mathbf{u}^{k}\right|^{2} d \mathbf{x}+\frac{\tau}{2} \int_{\Omega \times \mathbb{S}}\left|\nabla_{\mathbf{n}} f^{k}\right|^{2}\left(\digamma^{L}\right)^{\prime \prime}\left(f^{k}+\alpha\right) d \mathbf{n} d \mathbf{x} .
\end{aligned}
$$

It follows from Lemma 2.1 that

$$
J_{3,2}=\tau \int_{\Omega \times \mathbb{S}}(2 \mathbf{n} \otimes \mathbf{n}-\mathrm{Id}) f^{k}: \nabla_{\mathbf{x}} \mathbf{u}^{k} d \mathbf{n} d \mathbf{x} .
$$

Now we estimate $J_{4}$. First we divide it into four parts as follows:

$$
\begin{aligned}
J_{4}=- & \tau \int_{\Omega \times \mathbb{S}} E^{L}\left(f^{k}\right)(\operatorname{Id}+\mathbf{n} \otimes \mathbf{n}) \mathbf{e}_{2} \cdot \mathbf{e}_{2} d \mathbf{n} d \mathbf{x} \\
& -\tau \int_{\Omega \times \mathbb{S}}\left(\digamma^{L}\right)^{\prime \prime}\left(f^{k}+\alpha\right)(\operatorname{Id}+\mathbf{n} \otimes \mathbf{n}) \nabla_{\mathbf{x}} f^{k} \cdot \nabla_{\mathbf{x}} f^{k} d \mathbf{n} d \mathbf{x} \\
& -\tau \int_{\Omega \times \mathbb{S}}(\mathrm{Id}+\mathbf{n} \otimes \mathbf{n}) \nabla_{\mathbf{x}} f^{k} \cdot \mathbf{e}_{2} d \mathbf{n} d \mathbf{x} \\
& -\tau \int_{\Omega \times \mathbb{S}} E^{L}\left(f^{k}\right)\left(\digamma^{L}\right)^{\prime \prime}\left(f^{k}+\alpha\right)(\mathrm{Id}+\mathbf{n} \otimes \mathbf{n}) \mathbf{e}_{2} \cdot \nabla_{\mathbf{x}} f^{k} d \mathbf{n} d \mathbf{x} \\
=: & J_{4,1}+J_{4,2}+J_{4,3}+J_{4,4} .
\end{aligned}
$$

Because $\operatorname{Id}+\mathbf{n} \otimes \mathbf{n}$ is a positive definite matrix with smallest eigenvalue 1 , we have

$$
\begin{aligned}
J_{4,1}+J_{4,2} & \leq-\tau \int_{\Omega \times \mathbb{S}} E^{L}\left(f^{k}\right) d \mathbf{n} d \mathbf{x}-\tau \int_{\Omega \times \mathbb{S}}\left|\nabla_{\mathbf{x}} f^{k}\right|^{2}\left(\digamma^{L}\right)^{\prime \prime}\left(f^{k}+\alpha\right) d \mathbf{n} d \mathbf{x} \\
& \leq-\tau \int_{\Omega \times \mathbb{S}}\left|\nabla_{\mathbf{x}} f^{k}\right|^{2}\left(\digamma^{L}\right)^{\prime \prime}\left(f^{k}+\alpha\right) d \mathbf{n} d \mathbf{x} .
\end{aligned}
$$

It follows from Cauchy-Schwartz inequality that

$$
\begin{aligned}
J_{4,3} & \leq C \tau \int_{\Omega \times \mathbb{S}}\left|\nabla_{\mathbf{x}} f^{k}\right| d \mathbf{n} d \mathbf{x}=C \tau \int_{\Omega \times \mathbb{S}} \frac{\left|\nabla_{\mathbf{x}} f^{k}\right|}{\sqrt{f^{k}+\alpha}} \sqrt{f^{k}+\alpha} d \mathbf{n} d \mathbf{x} \\
& \leq C \tau \int_{\Omega \times \mathbb{S}}\left(f^{k}+\alpha\right) d \mathbf{n} d \mathbf{x}+\frac{\tau}{8} \int_{\Omega \times \mathbb{S}} \frac{\left|\nabla_{\mathbf{x}} f^{k}\right|^{2}}{f^{k}+\alpha} d \mathbf{n} d \mathbf{x} \\
& \leq C \alpha \tau+C \tau\left\|f^{k}\right\|_{L^{1}(\Omega \times \mathbb{S})}+\frac{\tau}{8} \int_{\Omega \times \mathbb{S}} \frac{\left|\nabla_{\mathbf{x}} f^{k}\right|^{2}}{f^{k}+\alpha} d \mathbf{n} d \mathbf{x} .
\end{aligned}
$$

Similar to (3.29) and (3.33), we deduce that

$$
J_{4,4}=-\tau \int_{\Omega \times \mathbb{S}}\left[E^{L}\left(f^{k}\right)\left(\digamma^{L}\right)^{\prime \prime}\left(f^{k}+\alpha\right)-1\right](\operatorname{Id}+\mathbf{n} \otimes \mathbf{n}) \mathbf{e}_{2} \cdot \nabla_{\mathbf{x}} f^{k} d \mathbf{n} d \mathbf{x}
$$




$$
\begin{aligned}
& -\tau \int_{\Omega \times \mathbb{S}}(\operatorname{Id}+\mathbf{n} \otimes \mathbf{n}) \mathbf{e}_{2} \cdot \nabla_{\mathbf{x}} f^{k} d \mathbf{n} d \mathbf{x} \\
& \leq C \alpha \tau+C \tau\left\|f^{k}\right\|_{L^{1}(\Omega \times \mathbb{S})}+\frac{\tau}{4} \int_{\Omega \times \mathbb{S}}\left|\nabla_{\mathbf{x}} f^{k}\right|^{2}\left(\digamma^{L}\right)^{\prime \prime}\left(f^{k}+\alpha\right) d \mathbf{n} d \mathbf{x} \\
& \quad+\frac{\tau}{8} \int_{\Omega \times \mathbb{S}} \frac{\left|\nabla_{\mathbf{x}} f^{k}\right|^{2}}{f^{k}+\alpha} d \mathbf{n} d \mathbf{x} .
\end{aligned}
$$

Consequently, (2.4) implies

$$
\begin{aligned}
J_{4} \leq- & \frac{\tau}{2} \int_{\Omega \times \mathbb{S}}\left|\nabla_{\mathbf{x}} f^{k}\right|^{2}\left(\digamma^{L}\right)^{\prime \prime}\left(f^{k}+\alpha\right) d \mathbf{n} d \mathbf{x} \\
& +C \alpha \tau+C \tau\left\|f^{k}\right\|_{L^{1}(\Omega \times \mathbb{S})}+\frac{\tau}{4} \int_{\Omega \times \mathbb{S}}\left|\nabla_{\mathbf{x}} f^{k}\right|^{2}\left[\frac{1}{f^{k}+\alpha}-\left(\digamma^{L}\right)^{\prime \prime}\left(f^{k}+\alpha\right)\right] d \mathbf{n} d \mathbf{x} \\
\leq- & \frac{\tau}{2} \int_{\Omega \times \mathbb{S}}\left|\nabla_{\mathbf{x}} f^{k}\right|^{2}\left(\digamma^{L}\right)^{\prime \prime}\left(f^{k}+\alpha\right) d \mathbf{n} d \mathbf{x}+C \alpha \tau+C \tau\left\|f^{k}\right\|_{L^{1}(\Omega \times \mathbb{S})}
\end{aligned}
$$

Taking $\mathbf{v}=\mathbf{u}^{k}$ in (3.5), one has

$$
\begin{aligned}
\tau \int_{\Omega}\left|\nabla_{\mathbf{x}} \mathbf{u}^{k}\right|^{2} d \mathbf{x} & =-\tau \int_{\Omega \times \mathbb{S}}(2 \mathbf{n} \otimes \mathbf{n}-\mathrm{Id}) f^{k}: \nabla_{\mathbf{x}} \mathbf{u}^{k} d \mathbf{n} d \mathbf{x}-\tau \int_{\Omega \times \mathbb{S}}\left(\mathbf{u}^{k} f^{k}\right) \cdot \mathbf{e}_{2} d \mathbf{n} d \mathbf{x} \\
& =-J_{3,2}-J_{2}
\end{aligned}
$$

Combining (3.27)-(3.36) and summing up, we have, by noting that $f^{0}=E^{L}\left(f_{0}\right)$, that

$$
\begin{aligned}
& \int_{\Omega \times \mathbb{S}} \digamma^{L}\left(f^{k}+\alpha\right) d \mathbf{n} d \mathbf{x}+\tau(1-C \alpha) \sum_{i=1}^{k} \int_{\Omega}\left|\nabla_{\mathbf{x}} \mathbf{u}^{i}\right|^{2} d \mathbf{x} \\
& +\frac{\tau}{2} \sum_{i=1}^{k} \int_{\Omega \times \mathbb{S}}\left(\left|\nabla_{\mathbf{x}} f^{i}\right|^{2}+\left|\nabla_{\mathbf{n}} f^{i}\right|^{2}\right)\left(\digamma^{L}\right)^{\prime \prime}\left(f^{i}+\alpha\right) d \mathbf{n} d \mathbf{x} \\
& \leq \int_{\Omega \times \mathbb{S}} \digamma^{L}\left(E^{L}\left(f_{0}\right)+\alpha\right) d \mathbf{n} d \mathbf{x}+\int_{\Omega \times \mathbb{S}}\left[E^{L}\left(f_{0}\right)-f^{k}\right] \mathbf{x} \cdot \mathbf{e}_{2} d \mathbf{n} d \mathbf{x} \\
& \quad+C \tau \sum_{i=1}^{k}\left\|f^{i}\right\|_{L^{1}(\Omega \times \mathbb{S})}+C \alpha .
\end{aligned}
$$

Thus it follows from (2.3), (2.4), (2.7), and (3.25) that

$$
\begin{gathered}
\int_{\Omega \times \mathbb{S}} \digamma\left(f^{k}+\alpha\right) d \mathbf{n} d \mathbf{x}+\tau(1-C \alpha) \sum_{i=1}^{k} \int_{\Omega}\left|\nabla_{\mathbf{x}} \mathbf{u}^{i}\right|^{2} d \mathbf{x} \\
+\frac{\tau}{2} \sum_{i=1}^{k} \int_{\Omega \times \mathbb{S}}\left(\frac{\left|\nabla_{\mathbf{x}} f^{i}\right|^{2}}{f^{i}+\alpha}+\frac{\left|\nabla_{\mathbf{n}} f^{i}\right|^{2}}{f^{i}+\alpha}\right) d \mathbf{n} d \mathbf{x} \\
\leq \int_{\Omega \times \mathbb{S}}\left[\alpha+\frac{\alpha^{2}}{2}+\digamma\left(f_{0}+\alpha\right)\right] d \mathbf{n} d \mathbf{x}+C\left\|f_{0}\right\|_{L^{1}(\Omega \times \mathbb{S})}+C \alpha .
\end{gathered}
$$

Choosing sufficiently small $\alpha>0$ and then performing $\alpha \rightarrow 0$, one finishes the proof by applying Lebesgue's dominated convergence theorem and Fatou's lemma. 


\section{3. $L^{2}(\Omega \times \mathbb{S})$ and time regularity estimates, uniform in $\tau$.}

Lemma 3.7. For any fixed $T>0$, we might as well let $N=T / \tau$ (otherwise let $N=[T / \tau]+1)$, then

$$
\sup _{1 \leq k \leq N}\left\|f^{k}\right\|_{L^{2}(\Omega \times \mathbb{S})}^{2}+\tau \sum_{k=1}^{N}\left[\left\|\nabla_{\mathbf{x}} f^{i}\right\|_{L^{2}(\Omega \times \mathbb{S})}^{2}+\left\|\nabla_{\mathbf{n}} f^{i}\right\|_{L^{2}(\Omega \times \mathbb{S})}^{2}\right] \leq C(T) .
$$

Proof. Denote $L:=\tau^{-\frac{1}{4}}$. Taking $\varphi=f^{k}$ in (3.6) and performing a procedure similar to $(3.21)$, we have

$$
\begin{aligned}
& \frac{1}{2} \int_{\Omega \times \mathbb{S}}\left|f^{k}\right|^{2} d \mathbf{n} d \mathbf{x}+\tau \int_{\Omega \times \mathbb{S}}\left(\left|\nabla_{\mathbf{x}} f^{k}\right|^{2}+\left|\nabla_{\mathbf{n}} f^{k}\right|^{2}\right) d \mathbf{n} d \mathbf{x} \\
= & \frac{1}{2} \int_{\Omega \times \mathbb{S}}\left|f^{k-1}\right|^{2} d \mathbf{n} d \mathbf{x}-\tau \int_{\Omega \times \mathbb{S}}\left[(\mathrm{Id}+\mathbf{n} \otimes \mathbf{n}) \mathbf{e}_{2}\right] E^{L}\left(f^{k}\right) \cdot \nabla_{\mathbf{x}} f^{k} d \mathbf{n} d \mathbf{x} \\
& \quad+\tau \int_{\Omega \times \mathbb{S}}\left[(\mathrm{Id}-\mathbf{n} \otimes \mathbf{n}) \nabla_{\mathbf{x}} \mathbf{u}^{k} \mathbf{n}\right] E^{L}\left(f^{k}\right) \cdot \nabla_{\mathbf{n}} f^{k} d \mathbf{n} d \mathbf{x} \\
= & \frac{1}{2} \int_{\Omega \times \mathbb{S}}\left|f^{k-1}\right|^{2} d \mathbf{n} d \mathbf{x}+P_{1}+P_{2}
\end{aligned}
$$

and

$$
P_{1} \leq C \tau \int_{\Omega \times \mathbb{S}}\left|f^{k}\right|\left|\nabla_{\mathbf{x}} f^{k}\right| d \mathbf{n} d \mathbf{x} \leq \frac{\tau}{4} \int_{\Omega \times \mathbb{S}}\left|\nabla_{\mathbf{x}} f^{k}\right|^{2} d \mathbf{n} d \mathbf{x}+C \tau \int_{\Omega \times \mathbb{S}}\left|f^{k}\right|^{2} d \mathbf{n} d \mathbf{x} .
$$

It follows from Lemma 2.1, (2.2), and Hölder's inequality that

$$
\begin{aligned}
P_{2} & =\tau \int_{\Omega \times \mathbb{S}}\left[(\mathrm{Id}-\mathbf{n} \otimes \mathbf{n}) \nabla_{\mathbf{x}} \mathbf{u}^{k} \mathbf{n}\right] \cdot \nabla_{\mathbf{n}} G^{L}\left(f^{k}\right) d \mathbf{n} d \mathbf{x} \\
& =\tau \int_{\Omega \times \mathbb{S}}(2 \mathbf{n} \otimes \mathbf{n}-\mathrm{Id}) G^{L}\left(f^{k}\right): \nabla_{\mathbf{x}} \mathbf{u}^{k} d \mathbf{n} d \mathbf{x} \leq C \tau \int_{\Omega \times \mathbb{S}}\left|G^{L}\left(f^{k}\right) \| \nabla_{\mathbf{x}} \mathbf{u}^{k}\right| d \mathbf{n} d \mathbf{x} \\
& \leq C \tau \int_{\Omega \times \mathbb{S}}\left|f^{k}\right|^{2}\left|\nabla_{\mathbf{x}} \mathbf{u}^{k}\right| d \mathbf{n} d \mathbf{x} \leq C \tau\left\|\nabla_{\mathbf{x}} \mathbf{u}^{k}\right\|_{L^{2}(\Omega)}\left\|f^{k}\right\|_{L^{4}\left(\Omega ; L^{2}(\mathbb{S})\right)}^{2} .
\end{aligned}
$$

Applying the Gagliardo-Nirenberg inequality, we have that

$$
\begin{aligned}
\left\|f^{k}\right\|_{L^{4}\left(\Omega ; L^{2}(\mathbb{S})\right)}^{2} & \leq C\left\|f^{k}\right\|_{H^{1}\left(\Omega ; L^{2}(\mathbb{S})\right)}\left\|f^{k}\right\|_{L^{2}\left(\Omega ; L^{2}(\mathbb{S})\right)} \\
& \leq C\left(\left\|\nabla_{\mathbf{x}} f^{k}\right\|_{L^{2}(\Omega \times \mathbb{S})}\left\|f^{k}\right\|_{L^{2}(\Omega \times \mathbb{S})}+\left\|f^{k}\right\|_{L^{2}(\Omega \times \mathbb{S})}^{2}\right) .
\end{aligned}
$$

Therefore by Cauchy-Schwartz inequality, one has

$$
P_{2} \leq \frac{\tau}{4}\left\|\nabla_{\mathbf{x}} f^{k}\right\|_{L^{2}(\Omega \times \mathbb{S})}^{2}+C \tau\left(\left\|\nabla_{\mathbf{x}} \mathbf{u}^{k}\right\|_{L^{2}(\Omega)}^{2}+\left\|\nabla_{\mathbf{x}} \mathbf{u}^{k}\right\|_{L^{2}(\Omega)}\right)\left\|f^{k}\right\|_{L^{2}(\Omega \times \mathbb{S})}^{2} .
$$

Combining (3.39), (3.40), and (3.43), then summing up, we deduce that

$$
\begin{aligned}
& \left\|f^{k}\right\|_{L^{2}(\Omega \times \mathbb{S})}^{2}+\sum_{i=1}^{k}\left\|f^{i}-f^{i-1}\right\|_{L^{2}(\Omega \times \mathbb{S})}^{2}+\tau \sum_{i=1}^{k}\left(\left\|\nabla_{\mathbf{x}} f^{i}\right\|_{L^{2}(\Omega \times \mathbb{S})}^{2}+\left\|\nabla_{\mathbf{n}} f^{i}\right\|_{L^{2}(\Omega \times \mathbb{S})}^{2}\right) \\
\leq & \left\|f^{0}\right\|_{L^{2}(\Omega \times \mathbb{S})}^{2}+C \tau \sum_{i=1}^{k}\left(\left\|\nabla_{\mathbf{x}} \mathbf{u}^{i}\right\|_{L^{2}(\Omega)}^{2}+1\right)\left\|f^{i}\right\|_{L^{2}(\Omega \times \mathbb{S})}^{2}
\end{aligned}
$$


It follows from $(3.22)$ and $0 \leq f^{0}=E^{L}\left(f_{0}\right) \leq f_{0}$ that

$$
\begin{aligned}
\left\|f^{k}\right\|_{L^{2}(\Omega \times \mathbb{S})}^{2} & \leq\left\|f_{0}\right\|_{L^{2}(\Omega \times \mathbb{S})}^{2}+2 C \tau \sum_{i=1}^{k}\left(\left\|\nabla_{\mathbf{x}} \mathbf{u}^{i}\right\|_{L^{2}(\Omega)}^{2}+1\right)\left\|f^{i-1}\right\|_{L^{2}(\Omega \times \mathbb{S})}^{2} \\
& =\left\|f_{0}\right\|_{L^{2}(\Omega \times \mathbb{S})}^{2}+2 C \tau \sum_{i=0}^{k-1}\left(\left\|\nabla_{\mathbf{x}} \mathbf{u}^{i+1}\right\|_{L^{2}(\Omega)}^{2}+1\right)\left\|f^{i}\right\|_{L^{2}(\Omega \times \mathbb{S})}^{2} .
\end{aligned}
$$

Therefore (3.45) and Lemma 3.6 imply (3.38) by employing the discrete Gronwall inequality. This finishes the proof of Lemma 3.7.

The next result follows from the regularity of Stokes' equation (see [26], p.35, Proposition 2.3).

Lemma 3.8. For any $k \in \mathbb{N}$,

$$
\left\|\nabla_{\mathbf{x}} \mathbf{u}^{k}\right\|_{L^{2}(\Omega)} \leq C\left\|f^{k}\right\|_{L^{2}\left(\Omega ; L^{1}(\mathbb{S})\right)} ;\left\|\mathbf{u}^{k}\right\|_{H^{2}(\Omega)} \leq C\left\|\nabla_{\mathbf{x}} f^{k}\right\|_{L^{2}\left(\Omega ; L^{1}(\mathbb{S})\right)} .
$$

Definition 3.9. Define the piecewise function in $t$ by

$$
f_{\tau}(t, \cdot, \cdot):=f^{k}(\cdot, \cdot), t \in((k-1) \tau, k \tau], k \in \mathbb{N},
$$

and the difference quotient of size $\tau$ by

$$
\partial_{t}^{\tau} f_{\tau}(t, \cdot, \cdot):=\frac{f^{k}(\cdot, \cdot)-f^{k-1}(\cdot, \cdot)}{\tau}, t \in((k-1) \tau, k \tau], k \in \mathbb{N} .
$$

Likewise, define $\mathbf{u}_{\tau}$.

Corollary 3.10. For any fixed $T>0$,

$$
\begin{aligned}
\left\|\mathbf{u}_{\tau}\right\|_{L^{\infty}(0, T ; \boldsymbol{V}) \cap L^{2}\left(0, T ; H^{2}(\Omega)\right)} & \leq C(T), \\
\left\|f_{\tau}\right\|_{L^{\infty}\left(0, T ; L^{2}(\Omega \times \mathbb{S})\right) \cap L^{2}\left(0, T ; H^{1}(\Omega \times \mathbb{S})\right)} & \leq C(T) .
\end{aligned}
$$

Proof. We can deduce (3.47)-(3.48) directly from Lemma 3.6-3.8.

Lemma 3.11. For any fixed $T>0$,

$$
\left\|\partial_{t}^{\tau} f_{\tau}\right\|_{L^{2}\left(0, T ;\left(H^{1}(\Omega \times \mathbb{S})\right)^{\prime}\right)} \leq C(T) .
$$

Proof. We deduce from (3.6) and the Poincaré inequality that

$$
\begin{aligned}
& \left\|\frac{f^{k}-f^{k-1}}{\tau}\right\|_{\left(H^{1}(\Omega \times \mathbb{S})\right)^{\prime}} \\
\leq & C\left[\left\|f^{k}\right\|_{H^{1}(\Omega \times \mathbb{S})}+\left(\left\|\mathbf{u}^{k}\right\|_{L^{4}(\Omega)}+\left\|\nabla_{\mathbf{x}} \mathbf{u}^{k}\right\|_{L^{4}(\Omega)}\right)\left\|f^{k}\right\|_{L^{4}\left(\Omega ; L^{2}(\mathbb{S})\right)}\right] \\
\leq & C\left[\left\|f^{k}\right\|_{H^{1}(\Omega \times \mathbb{S})}+\left\|\nabla_{\mathbf{x}} \mathbf{u}^{k}\right\|_{L^{4}(\Omega)}\left\|f^{k}\right\|_{L^{4}\left(\Omega ; L^{2}(\mathbb{S})\right)}\right] .
\end{aligned}
$$

For any fixed $T>0$, we might as well let $N=T / \tau$ (otherwise let $N=[T / \tau]+1$ ), so that 


$$
\begin{aligned}
& \left\|\partial_{t}^{\tau} f_{\tau}\right\|_{L^{2}\left(0, T ;\left(H^{1}(\Omega \times \mathbb{S})\right)^{\prime}\right)} \\
= & \left(\tau \sum_{k=1}^{N}\left\|\frac{f^{k}-f^{k-1}}{\tau}\right\|_{\left(H^{1}(\Omega \times \mathbb{S})\right)^{\prime}}^{2}\right)^{1 / 2} \\
\leq & C\left(\tau \sum_{k=1}^{N}\left[\left\|f^{k}\right\|_{H^{1}(\Omega \times \mathbb{S})}+\left\|\nabla_{\mathbf{x}} \mathbf{u}^{k}\right\|_{L^{4}(\Omega)}\left\|f^{k}\right\|_{L^{4}\left(\Omega ; L^{2}(\mathbb{S})\right)}\right]\right)^{1 / 2} \\
= & C\left(\sum_{k=1}^{N} \int_{(k-1) \tau}^{k \tau}\left[\left\|f_{\tau}\right\|_{H^{1}(\Omega \times \mathbb{S})}+\left\|\nabla_{\mathbf{x}} \mathbf{u}_{\tau}\right\|_{L^{4}(\Omega)}\left\|f_{\tau}\right\|_{L^{4}\left(\Omega ; L^{2}(\mathbb{S})\right)}\right]\right)^{1 / 2} \\
\leq & C\left(\left\|f_{\tau}\right\|_{L^{2}\left(0, T ; H^{1}(\Omega \times \mathbb{S})\right)}+\left\|f_{\tau}\right\|_{L^{4}\left(0, T ; L^{4}\left(\Omega ; L^{2}(\mathbb{S})\right)\right)}\left\|\nabla_{\mathbf{x}} \mathbf{u}_{\tau}\right\|_{L^{4}\left(0, T ; L^{4}(\Omega)\right)}\right) .
\end{aligned}
$$

It follows from the Gagliardo-Nirenberg inequality that

$$
\left\|\nabla_{\mathbf{x}} \mathbf{u}_{\tau}\right\|_{L^{4}(\Omega)} \leq C\left\|\mathbf{u}_{\tau}\right\|_{H^{2}(\Omega)}^{1 / 2}\left\|\nabla_{\mathbf{x}} \mathbf{u}_{\tau}\right\|_{L^{2}(\Omega)}^{1 / 2}
$$

and hence by Hölder's inequality that

$$
\left\|\nabla_{\mathbf{x}} \mathbf{u}_{\tau}\right\|_{L^{4}\left(0, T ; L^{4}(\Omega)\right)} \leq C\left\|\mathbf{u}_{\tau}\right\|_{L^{2}\left(0, T ; H^{2}(\Omega)\right)}^{1 / 2}\left\|\nabla_{\mathbf{x}} \mathbf{u}_{\tau}\right\|_{L^{\infty}\left(0, T ; L^{2}(\Omega)\right)}^{1 / 2} .
$$

Now we estimate $\left\|f_{\tau}\right\|_{L^{4}\left(0, T ; L^{4}\left(\Omega ; L^{2}(\mathbb{S})\right)\right)}$. Applying the same discussion as for (2.12), we deduce from the Gagliardo-Nirenberg inequality and Hölder's inequality that

$$
\left\|f_{\tau}\right\|_{L^{4}\left(0, T ; L^{4}\left(\Omega ; L^{2}(\mathbb{S})\right)\right)} \leq\left\|f_{\tau}\right\|_{L^{2}\left(0, T ; H^{1}(\Omega \times \mathbb{S})\right)}^{1 / 2}\left\|f_{\tau}\right\|_{L^{\infty}\left(0, T ; L^{2}(\Omega \times \mathbb{S})\right)}^{1 / 2} .
$$

Combining (3.51)-(3.53), we deduce from Corollary 3.10 that (3.49) holds. This ends the proof of Lemma 3.11 .

\subsection{Convergence and proof of Theorem 3.1.}

Proposition 3.12. As $\tau \rightarrow 0$, there exist a subsequence of $\left\{\left(\mathbf{u}_{\tau}, f_{\tau}\right)\right\}_{0<\tau \ll 1}$, not relabeled, and a pair of functions $(\mathbf{u}, f)$ satisfying (2.8)-(2.9), (3.1)-(3.3), and (3.4) such that

$$
\begin{aligned}
\mathbf{u}_{\tau} \rightarrow \mathbf{u} & \text { in } L^{2}(0, \infty ; \boldsymbol{V}), \\
\sqrt{f_{\tau} *} \sqrt{f} & \text { in } L^{\infty}\left(0, \infty ; L^{2}(\Omega \times \mathbb{S})\right), \\
\sqrt{f_{\tau}} \rightarrow \sqrt{f} & \text { in } L^{2}\left(0, \infty ; H^{1}(\Omega \times \mathbb{S})\right),
\end{aligned}
$$

and for any $T>0$,

$$
\begin{aligned}
\mathbf{u}_{\tau} \stackrel{*}{\rightarrow} \mathbf{u} & \text { in } L^{\infty}(0, T ; \boldsymbol{V}), \\
\mathbf{u}_{\tau} \rightarrow \mathbf{u} & \text { in } L^{2}\left(0, T ; \boldsymbol{V}^{2}\right), \\
f_{\tau} \stackrel{*}{ } f & \text { in } L^{\infty}\left(0, T ; L^{2}(\Omega \times \mathbb{S})\right), \\
f_{\tau} \rightarrow f & \text { in } L^{2}\left(0, T ; H^{1}(\Omega \times \mathbb{S})\right), \\
f_{\tau} \rightarrow f & \text { in } L^{2}((0, T) \times \Omega \times \mathbb{S}), \\
E^{\tau^{-\frac{1}{4}}}\left(f_{\tau}\right) \rightarrow f & \text { in } L^{2}((0, T) \times \Omega \times \mathbb{S}) .
\end{aligned}
$$


Proof. Applying Lemma 3.5-3.8 and Corollary 3.10, we deduce that there exist a subsequence and a pair of functions $(\mathbf{u}, f)$ which satisfy (2.8)-(2.9), (3.1)-(3.3), and (3.54)-(3.60).

For any fixed $T>0$, we might as well let $N=T / \tau$ (otherwise let $N=[T / \tau]+1)$. Then we have from (3.38) and (3.49) that

$$
\left\|\tau_{\tau} f_{\tau}-f_{\tau}\right\|_{L^{2}\left(0, T-\tau ;\left(H^{1}(\Omega \times \mathbb{S})\right)^{\prime}\right)}^{2}=\tau \sum_{k=1}^{N-1}\left\|f^{k+1}-f^{k}\right\|_{\left(H^{1}(\Omega \times \mathbb{S})\right)^{\prime}}^{2} \leq C \tau^{2},
$$

where $\tau_{\tau} u_{\tau}(t):=u_{\tau}(t+\tau)$. Employing Theorem 1 in [11], we obtain (3.61) from (3.63), (3.48), and $H^{1}(\Omega \times \mathbb{S}) \hookrightarrow \hookrightarrow L^{2}(\Omega \times \mathbb{S})$. Then we have from $E^{\tau^{-\frac{1}{4}}} \in C^{0,1}(\mathbb{R})$ with Lipschitz coefficient 1 that

$$
\begin{aligned}
& \left\|E^{\tau^{-\frac{1}{4}}}\left(f_{\tau}\right)-f\right\|_{L^{2}((0, T) \times \Omega \times \mathbb{S})} \\
\leq & \left\|E^{\tau^{-\frac{1}{4}}}\left(f_{\tau}\right)-E^{\tau^{-\frac{1}{4}}}(f)\right\|_{L^{2}((0, T) \times \Omega \times \mathbb{S})}+\left\|E^{\tau^{-\frac{1}{4}}}(f)-f\right\|_{L^{2}((0, T) \times \Omega \times \mathbb{S})} \\
\leq & \left\|f_{\tau}-f\right\|_{L^{2}((0, T) \times \Omega \times \mathbb{S})}+\left\|E^{\tau^{-\frac{1}{4}}}(f)-f\right\|_{L^{2}((0, T) \times \Omega \times \mathbb{S})} .
\end{aligned}
$$

Moreover, employing Lebesgue' dominated convergence theorem, one deduces from (2.6) and $0 \leq E^{\tau^{-\frac{1}{4}}}(f) \leq f$ that

$$
\left\|E^{\tau^{-\frac{1}{4}}}(f)-f\right\|_{L^{2}((0, T) \times \Omega \times \mathbb{S})} \rightarrow 0 \text { as } \tau \rightarrow 0 .
$$

Then (3.65) and (3.64) imply (3.62).

In light of the weakly lower semi-continuity of norm, we obtain the energy inequality (3.4) directly from (3.26) and the convergent results (3.54)-(3.56) and (3.61). This ends the proof of Proposition 3.12.

Next we shall prove Theorem 3.1. We need to establish the convergence of the discrete derivatives $\partial_{t}^{\tau} f_{\tau}$ as well as their weak integral. These follow from the time regularity estimate (Lemma 3.11) of $\partial_{t}^{\tau} f_{\tau}$ and its convergence to $\partial_{t} f$ in the sense of distribution.

Proof. [Proof of Theorem 3.1.] We only need to show that for any fixed $T>0,(2.10)$ (2.11) hold with time interval $[0, \infty)$ replaced by $[0, T)$ and test functions replaced by $\mathbf{v} \in L^{2}(0, T ; \boldsymbol{V})$ and $\varphi \in C^{\infty}([0, T) \times \bar{\Omega} \times \mathbb{S})$. Indeed, in view of Definition 3.3, the weak approximation form of (3.5)-(3.6) reads: for any $\mathbf{v} \in L^{2}(0, T ; \boldsymbol{V})$,

$$
\begin{aligned}
& \int_{0}^{T} \int_{\Omega} \nabla_{\mathbf{x}} \mathbf{u}_{\tau}: \nabla_{\mathbf{x}} \mathbf{v} d \mathbf{x} d t \\
= & -\int_{0}^{T} \int_{\Omega \times \mathbb{S}}(2 \mathbf{n} \otimes \mathbf{n}-\mathrm{Id}) f_{\tau}: \nabla_{\mathbf{x}} \mathbf{v} d \mathbf{n} d \mathbf{x} d t-\int_{0}^{T} \int_{\Omega \times \mathbb{S}} f_{\tau} \mathbf{e}_{2} \cdot \mathbf{v} d \mathbf{n} d \mathbf{x} d t
\end{aligned}
$$

and for any $\varphi \in C^{\infty}([0, T) \times \bar{\Omega} \times \mathbb{S})$,

$$
\begin{aligned}
& \int_{0}^{T} \int_{\Omega \times \mathbb{S}} \partial_{t}^{\tau} f_{\tau} \varphi d \mathbf{n} d \mathbf{x} d t-\int_{0}^{T} \int_{\Omega \times \mathbb{S}}\left(\mathbf{u}_{\tau} f_{\tau}\right) \cdot \nabla_{\mathbf{x}} \varphi d \mathbf{n} d \mathbf{x} d t \\
& \quad+\int_{0}^{T} \int_{\Omega \times \mathbb{S}} \nabla_{\mathbf{n}} f_{\tau} \cdot \nabla_{\mathbf{n}} \varphi d \mathbf{n} d \mathbf{x} d t
\end{aligned}
$$




$$
\begin{aligned}
= & \int_{0}^{T} \int_{\Omega \times \mathbb{S}}\left[(\mathrm{Id}-\mathbf{n} \otimes \mathbf{n}) \nabla_{\mathbf{x}} \mathbf{u}_{\tau} \mathbf{n}\right] E^{\tau^{-\frac{1}{4}}}\left(f_{\tau}\right) \cdot \nabla_{\mathbf{n}} \varphi d \mathbf{n} d \mathbf{x} d t \\
& -\int_{0}^{T} \int_{\Omega \times \mathbb{S}}(\operatorname{Id}+\mathbf{n} \otimes \mathbf{n})\left(\mathbf{e}_{2} E^{\tau^{-\frac{1}{4}}}\left(f_{\tau}\right)+\nabla_{\mathbf{x}} f_{\tau}\right) \cdot \nabla_{\mathbf{x}} \varphi d \mathbf{n} d \mathbf{x} d t .
\end{aligned}
$$

We first claim that as $\tau \rightarrow 0$,

$$
\begin{aligned}
& \int_{0}^{T} \int_{\Omega \times \mathbb{S}} \partial_{t}^{\tau} f_{\tau} \varphi d \mathbf{n} d \mathbf{x} d t \rightarrow-\int_{0}^{T} \int_{\Omega \times \mathbb{S}} f \partial_{t} \varphi d \mathbf{n} d \mathbf{x} d t-\int_{\Omega \times \mathbb{S}} f_{0}(\mathbf{x}, \mathbf{n}) \varphi(0, \mathbf{x}, \mathbf{n}) d \mathbf{n} d \mathbf{x} \\
& \partial_{t}^{\tau} f_{\tau} \rightarrow \partial_{t} f \text { in } L^{2}\left(0, T ;\left(H^{1}(\Omega \times \mathbb{S})\right)^{\prime}\right) .
\end{aligned}
$$

Indeed,

$$
\begin{aligned}
& \int_{0}^{T} \int_{\Omega \times \mathbb{S}} \partial_{t}^{\tau} f_{\tau} \varphi d \mathbf{n} d \mathbf{x} d t \\
= & \int_{\tau}^{T} \int_{\Omega \times \mathbb{S}} \frac{f_{\tau}(t)-f_{\tau}(t-\tau)}{\tau} \varphi d \mathbf{n} d \mathbf{x} d t+\int_{0}^{\tau} \int_{\Omega \times \mathbb{S}} \frac{f_{\tau}(t)-f^{0}}{\tau} \varphi d \mathbf{n} d \mathbf{x} d t \\
= & \int_{0}^{T} \int_{\Omega \times \mathbb{S}} \frac{f_{\tau}(t)}{\tau} \varphi d \mathbf{n} d \mathbf{x} d t-\int_{0}^{T-\tau} \int_{\Omega \times \mathbb{S}} \frac{f_{\tau}(t)}{\tau} \varphi(t+\tau) d \mathbf{n} d \mathbf{x} d t \\
& -\int_{0}^{\tau} \int_{\Omega \times \mathbb{S}} \frac{E^{\tau^{-\frac{1}{4}}}\left(f_{0}\right)}{\tau} \varphi d \mathbf{n} d \mathbf{x} d t \\
= & \int_{T-\tau}^{T} \int_{\Omega \times \mathbb{S}} f_{\tau}(t) \frac{\varphi}{\tau} d \mathbf{n} d \mathbf{x} d t-\int_{0}^{T-\tau} \int_{\Omega \times \mathbb{S}} f_{\tau}(t) \frac{\varphi(t+\tau)-\varphi(t)}{\tau} d \mathbf{n} d \mathbf{x} d t \\
& -\int_{0}^{\tau} \int_{\Omega \times \mathbb{S}} E^{\tau^{-\frac{1}{4}}}\left(f_{0}\right) \frac{\varphi}{\tau} d \mathbf{n} d \mathbf{x} d t .
\end{aligned}
$$

Then

$$
\begin{aligned}
& \left|\int_{0}^{T} \int_{\Omega \times \mathbb{S}} \partial_{t}^{\tau} f_{\tau} \varphi d \mathbf{n} d \mathbf{x} d t+\int_{0}^{T} \int_{\Omega \times \mathbb{S}} f \partial_{t} \varphi d \mathbf{n} d \mathbf{x} d t+\int_{\Omega \times \mathbb{S}} f_{0}(\mathbf{x}, \mathbf{n}) \varphi(0, \mathbf{x}, \mathbf{n}) d \mathbf{n} d \mathbf{x}\right| \\
& \leq\left|\int_{T-\tau}^{T} \int_{\Omega \times \mathbb{S}}\left(f_{\tau} \frac{\varphi}{\tau}+f \partial_{t} \varphi\right) d \mathbf{n} d \mathbf{x} d t\right| \\
& \quad+\left|\int_{0}^{T-\tau} \int_{\Omega \times \mathbb{S}}\left(f \partial_{t} \varphi-f_{\tau} \frac{\varphi(t+\tau)-\varphi(t)}{\tau}\right) d \mathbf{n} d \mathbf{x} d t\right| \\
& \quad+\left|\int_{\Omega \times \mathbb{S}}\left[f_{0} \varphi(0)-E^{\tau^{-\frac{1}{4}}}\left(f_{0}\right) \int_{0}^{\tau} \frac{\varphi}{\tau} d t\right] d \mathbf{n} d \mathbf{x}\right|=: I_{1}+I_{2}+I_{3} .
\end{aligned}
$$

Thanks to $\varphi(T)=0$, we have from the mean value theorem of differentials that

$$
\begin{gathered}
I_{1} \leq \tau\left\|\partial_{t} \varphi\right\|_{L^{\infty}((0, T) \times \Omega \times \mathbb{S})}\left(\left\|f_{\tau}\right\|_{L^{\infty}\left(0, T ; L^{1}(\Omega \times \mathbb{S})\right)}+\|f\|_{L^{\infty}\left(0, T ; L^{1}(\Omega \times \mathbb{S})\right)}\right) \leq C \tau, \\
I_{2} \leq\left|\int_{0}^{T-\tau} \int_{\Omega \times \mathbb{S}} f\left(\partial_{t} \varphi-\frac{\varphi(t+\tau)-\varphi(t)}{\tau}\right) d \mathbf{n} d \mathbf{x} d t\right|
\end{gathered}
$$




$$
\begin{aligned}
& \quad+\left|\int_{0}^{T-\tau} \int_{\Omega \times \mathbb{S}}\left(f-f_{\tau}\right) \frac{\varphi(t+\tau)-\varphi(t)}{\tau} d \mathbf{n} d \mathbf{x} d t\right| \\
& \leq \tau\left\|\partial_{t t} \varphi\right\|_{L^{\infty}((0, T) \times \Omega \times \mathbb{S})}\|f\|_{L^{\infty}\left(0, T ; L^{1}(\Omega \times \mathbb{S})\right)} \\
& \quad+\left\|f-f_{\tau}\right\|_{L^{1}((0, T) \times \Omega \times \mathbb{S})}\left\|\partial_{t} \varphi\right\|_{L^{\infty}((0, T) \times \Omega \times \mathbb{S})} \\
& \leq C\left(\tau+\left\|f-f_{\tau}\right\|_{L^{1}((0, T) \times \Omega \times \mathbb{S})}\right) .
\end{aligned}
$$

It follows from the proof of (3.65) and the mean value theorem that

$$
I_{3} \leq \int_{\Omega \times \mathbb{S}}\left|f_{0}-E^{\tau^{-\frac{1}{4}}}\left(f_{0}\right)\right||\varphi(0)| d \mathbf{n} d \mathbf{x}+\int_{\Omega \times \mathbb{S}} E^{\tau^{-\frac{1}{4}}}\left(f_{0}\right)\left|\varphi(0)-\frac{1}{\tau} \int_{0}^{\tau} \varphi(t) d t\right| d \mathbf{n} d \mathbf{x} \rightarrow 0 .
$$

Therefore (3.68) is proved. Moreover, if we take $\varphi \in C_{0}^{\infty}((0, T) \times \bar{\Omega} \times \mathbb{S})$, then (3.68) implies

$$
\partial_{t}^{\tau} f_{\tau} \rightarrow \partial_{t} f \text { in } \mathscr{D}^{\prime}\left((0, T) ;\left(C^{1}(\bar{\Omega} \times \mathbb{S})\right)^{\prime}\right) .
$$

Then (3.70) and Lemma 3.11 yield (3.69).

Combining Proposition 3.12, Lemma 3.6, and Corollary 3.10, we obtain Theorem 3.1 .

\section{Uniqueness}

TheOREM 4.1. The solution in Theorem 3.1 is unique.

Proof. We only need to prove uniqueness on $[0, T]$ for any $T>0$. Because $f \in$ $L^{2}\left(0, T ; H^{1}(\Omega \times \mathbb{S})\right) \cap H^{1}\left(0, T ;\left(H^{1}(\Omega \times \mathbb{S})\right)^{\prime}\right)$, we have $f \in C\left([0, T] ; L^{2}(\Omega \times \mathbb{S})\right)$ and

$$
\frac{d}{d t}\|f\|_{L^{2}(\Omega \times \mathbb{S})}^{2}=2\left\langle\partial_{t} f, f\right\rangle
$$

where $\langle\cdot, \cdot\rangle$ denotes the dual product between $H^{1}(\Omega \times \mathbb{S})$ and its dual $\left(H^{1}(\Omega \times \mathbb{S})\right)^{\prime}$ (see [26], p.260, Lemma 1.2). Suppose $\left(\mathbf{u}_{1}, f_{1}\right)$ and $\left(\mathbf{u}_{2}, f_{2}\right)$ are both solutions of (2.10)-(2.11). That is, for a.e. $t \in[0, T]$ and $i=1,2$,

$$
\begin{aligned}
& \int_{\Omega} \nabla_{\mathbf{x}} \mathbf{u}_{i}: \nabla_{\mathbf{x}} \mathbf{v} d \mathbf{x}=-\int_{\Omega \times \mathbb{S}}(2 \mathbf{n} \otimes \mathbf{n}-\mathrm{Id}) f_{i}: \nabla_{\mathbf{x}} \mathbf{v} d \mathbf{n} d \mathbf{x}-\int_{\Omega \times \mathbb{S}} f_{i} \mathbf{e}_{2} \cdot \mathbf{v} d \mathbf{n} d \mathbf{x}, \forall v \in \boldsymbol{V}, \\
& \left\langle\partial_{t} f_{i}, \varphi\right\rangle-\left(\mathbf{u}_{i} f_{i}, \nabla_{\mathbf{x}} \varphi\right)+\left(\nabla_{\mathbf{n}} f_{i}, \nabla_{\mathbf{n}} \varphi\right) \\
& =\left((\operatorname{Id}-\mathbf{n} \otimes \mathbf{n}) \nabla_{\mathbf{x}} \mathbf{u}_{i} \mathbf{n} f_{i}, \nabla_{\mathbf{n}} \varphi\right)-\left((\operatorname{Id}+\mathbf{n} \otimes \mathbf{n})\left(\mathbf{e}_{2} f_{i}+\nabla_{\mathbf{x}} f_{i}\right), \nabla_{\mathbf{x}} \varphi\right), \forall \varphi \in H^{1}(\Omega \times \mathbb{S}),
\end{aligned}
$$

and

$$
\left.f_{i}\right|_{t=0}=f_{0} \text { a.e. on } \Omega \times \mathbb{S} \text {. }
$$

We have from Corollary 3.10 and Proposition 3.12 that

$$
\left\|\mathbf{u}_{i}\right\|_{L^{\infty}\left(0, T ; H^{1}(\Omega)\right) \cap L^{2}\left(0, T ; H^{2}(\Omega)\right)}+\left\|f_{i}\right\|_{L^{\infty}\left(0, T ; L^{2}(\Omega \times \mathbb{S})\right) \cap L^{2}\left(0, T ; H^{1}(\Omega \times \mathbb{S})\right)} \leq C(T) .
$$

By substraction and then setting $\mathbf{v}=\mathbf{u}_{1}-\mathbf{u}_{2}$ in (4.2), we have by the CauchySchwartz inequality and the regularity of weak solution to the Stokes' equation (see [26], p.35, Proposition 2.3) that for a.e. $t \in[0, T]$,

$$
\left\|\nabla_{\mathbf{x}} \mathbf{u}_{1}-\nabla_{\mathbf{x}} \mathbf{u}_{2}\right\|_{L^{2}(\Omega)} \leq C\left\|f_{1}-f_{2}\right\|_{L^{2}(\Omega \times \mathbb{S})},
$$




$$
\left\|\mathbf{u}_{1}-\mathbf{u}_{2}\right\|_{H^{2}(\Omega)} \leq C\left\|\nabla_{\mathbf{x}} f_{1}-\nabla_{\mathbf{x}} f_{2}\right\|_{L^{2}(\Omega \times \mathbb{S})} .
$$

Substracting and then setting $\varphi=f_{1}-f_{2}$ in (4.3), we have, by noting (4.1), that for a.e. $t \in[0, T]$,

$$
\begin{aligned}
& \frac{1}{2} \frac{d}{d t}\left\|f_{1}-f_{2}\right\|_{L^{2}(\Omega \times \mathbb{S})}^{2}+\left\|\nabla_{\mathbf{n}} f_{1}-\nabla_{\mathbf{n}} f_{2}\right\|_{L^{2}(\Omega \times \mathbb{S})}^{2} \\
= & \left(\mathbf{u}_{1} f_{1}-\mathbf{u}_{2} f_{2}, \nabla_{\mathbf{x}} f_{1}-\nabla_{\mathbf{x}} f_{2}\right)+\left((\mathrm{Id}-\mathbf{n} \otimes \mathbf{n})\left(\nabla_{\mathbf{x}} \mathbf{u}_{1} \mathbf{n} f_{1}-\nabla_{\mathbf{x}} \mathbf{u}_{2} \mathbf{n} f_{2}\right), \nabla_{\mathbf{n}} f_{1}-\nabla_{\mathbf{n}} f_{2}\right) \\
& \quad-\left((\operatorname{Id}+\mathbf{n} \otimes \mathbf{n}) \mathbf{e}_{2}\left(f_{1}-f_{2}\right), \nabla_{\mathbf{x}} f_{1}-\nabla_{\mathbf{x}} f_{2}\right) \\
& \quad-\left((\mathrm{Id}+\mathbf{n} \otimes \mathbf{n})\left(\nabla_{\mathbf{x}} f_{1}-\nabla_{\mathbf{x}} f_{2}\right), \nabla_{\mathbf{x}} f_{1}-\nabla_{\mathbf{x}} f_{2}\right) \\
= & : Q_{1}+Q_{2}+Q_{3}+Q_{4} .
\end{aligned}
$$

We deduce from (4.5), (4.6), Hölder's inequality, the Gagliardo-Nirenberg inequality, the Poincaré inequality, and the Cauchy-Schwartz inequality that for a.e. $t \in[0, T]$,

$$
\begin{aligned}
Q_{1} & =\left(\left(\mathbf{u}_{1}-\mathbf{u}_{2}\right) f_{1}, \nabla_{\mathbf{x}} f_{1}-\nabla_{\mathbf{x}} f_{2}\right)+\left(\mathbf{u}_{2}\left(f_{1}-f_{2}\right), \nabla_{\mathbf{x}} f_{1}-\nabla_{\mathbf{x}} f_{2}\right) \\
= & \left(\left(\mathbf{u}_{1}-\mathbf{u}_{2}\right) f_{1}, \nabla_{\mathbf{x}} f_{1}-\nabla_{\mathbf{x}} f_{2}\right) \\
\leq & \left\|\mathbf{u}_{1}-\mathbf{u}_{2}\right\|_{L^{4}(\Omega)}\left\|f_{1}\right\|_{L^{4}\left(\Omega ; L^{2}(\mathbb{S})\right)}\left\|\nabla_{\mathbf{x}} f_{1}-\nabla_{\mathbf{x}} f_{2}\right\|_{L^{2}(\Omega \times \mathbb{S})} \\
\leq & C\left\|\nabla_{\mathbf{x}} \mathbf{u}_{1}-\nabla_{\mathbf{x}} \mathbf{u}_{2}\right\|_{L^{2}(\Omega)}^{1 / 2}\left\|\mathbf{u}_{1}-\mathbf{u}_{2}\right\|_{L^{2}(\Omega)}^{1 / 2} \\
& \quad \times\left\|f_{1}\right\|_{H^{1}\left(\Omega ; L^{2}(\mathbb{S})\right)}^{1 / 2}\left\|f_{1}\right\|_{L^{2}(\Omega \times \mathbb{S})}^{1 / 2}\left\|\nabla_{\mathbf{x}} f_{1}-\nabla_{\mathbf{x}} f_{2}\right\|_{L^{2}(\Omega \times \mathbb{S})} \\
& \leq C\left\|f_{1}-f_{2}\right\|_{L^{2}(\Omega \times \mathbb{S})}\left\|f_{1}\right\|_{H^{1}(\Omega \times \mathbb{S})}^{1 / 2}\left\|\nabla_{\mathbf{x}} f_{1}-\nabla_{\mathbf{x}} f_{2}\right\|_{L^{2}(\Omega \times \mathbb{S})} \\
\leq & \frac{1}{8}\left\|\nabla_{\mathbf{x}} f_{1}-\nabla_{\mathbf{x}} f_{2}\right\|_{L^{2}(\Omega \times \mathbb{S})}^{2}+C\left\|f_{1}-f_{2}\right\|_{L^{2}(\Omega \times \mathbb{S})}^{2}\left\|f_{1}\right\|_{H^{1}(\Omega \times \mathbb{S})} .
\end{aligned}
$$

We divide $Q_{2}$ into two parts:

$$
\begin{aligned}
Q_{2}= & \left((\mathrm{Id}-\mathbf{n} \otimes \mathbf{n})\left(\nabla_{\mathbf{x}} \mathbf{u}_{1}-\nabla_{\mathbf{x}} \mathbf{u}_{2}\right) \mathbf{n} f_{1}, \nabla_{\mathbf{n}} f_{1}-\nabla_{\mathbf{n}} f_{2}\right) \\
& +\left((\mathrm{Id}-\mathbf{n} \otimes \mathbf{n}) \nabla_{\mathbf{x}} \mathbf{u}_{2} \mathbf{n}\left(f_{1}-f_{2}\right), \nabla_{\mathbf{n}} f_{1}-\nabla_{\mathbf{n}} f_{2}\right) \\
= & : Q_{2,1}+Q_{2,2} .
\end{aligned}
$$

Similar to (4.9), in light of (4.5)-(4.7), we have that for a.e. $t \in[0, T]$,

$$
\begin{aligned}
Q_{2,1} \leq & C\left\|\nabla_{\mathbf{x}} \mathbf{u}_{1}-\nabla_{\mathbf{x}} \mathbf{u}_{2}\right\|_{L^{4}(\Omega)}\left\|f_{1}\right\|_{L^{4}\left(\Omega ; L^{2}(\mathbb{S})\right)}\left\|\nabla_{\mathbf{n}} f_{1}-\nabla_{\mathbf{n}} f_{2}\right\|_{L^{2}(\Omega \times \mathbb{S})} \\
\leq & C\left\|\mathbf{u}_{1}-\mathbf{u}_{2}\right\|_{H^{2}(\Omega)}^{1 / 2}\left\|\nabla_{\mathbf{x}} \mathbf{u}_{1}-\nabla_{\mathbf{x}} \mathbf{u}_{2}\right\|_{L^{2}(\Omega)}^{1 / 2} \\
& \times\left\|f_{1}\right\|_{H^{1}\left(\Omega ; L^{2}(\mathbb{S})\right)}^{1 / 2}\left\|f_{1}\right\|_{L^{2}(\Omega \times \mathbb{S})}^{1 / 2}\left\|\nabla_{\mathbf{n}} f_{1}-\nabla_{\mathbf{n}} f_{2}\right\|_{L^{2}(\Omega \times \mathbb{S})} \\
\leq & C\left\|\nabla_{\mathbf{x}} f_{1}-\nabla_{\mathbf{x}} f_{2}\right\|_{L^{2}(\Omega \times \mathbb{S})}^{1 / 2}\left\|f_{1}-f_{2}\right\|_{L^{2}(\Omega \times \mathbb{S})}^{1 / 2}\left\|f_{1}\right\|_{H^{1}(\Omega \times \mathbb{S})}^{1 / 2}\left\|\nabla_{\mathbf{n}} f_{1}-\nabla_{\mathbf{n}} f_{2}\right\|_{L^{2}(\Omega \times \mathbb{S})} \\
\leq & \frac{1}{2}\left\|\nabla_{\mathbf{n}} f_{1}-\nabla_{\mathbf{n}} f_{2}\right\|_{L^{2}(\Omega \times \mathbb{S})}^{2} \\
\quad & +C\left\|\nabla_{\mathbf{x}} f_{1}-\nabla_{\mathbf{x}} f_{2}\right\|_{L^{2}(\Omega \times \mathbb{S})}\left\|f_{1}-f_{2}\right\|_{L^{2}(\Omega \times \mathbb{S})}\left\|f_{1}\right\|_{H^{1}(\Omega \times \mathbb{S})} \\
\leq & \frac{1}{2}\left\|\nabla_{\mathbf{n}} f_{1}-\nabla_{\mathbf{n}} f_{2}\right\|_{L^{2}(\Omega \times \mathbb{S})}^{2}+\frac{1}{8}\left\|\nabla_{\mathbf{x}} f_{1}-\nabla_{\mathbf{x}} f_{2}\right\|_{L^{2}(\Omega \times \mathbb{S})}^{2} \\
& +C\left\|f_{1}-f_{2}\right\|_{L^{2}(\Omega \times \mathbb{S})}^{2}\left\|f_{1}\right\|_{H^{1}(\Omega \times \mathbb{S})}^{2} .
\end{aligned}
$$


It follows from Lemma 2.1, the Gagliardo-Nirenberg inequality, and (4.5) that for a.e. $t \in[0, T]$,

$$
\begin{aligned}
Q_{2,2} & =\frac{1}{2}\left((\mathrm{Id}-\mathbf{n} \otimes \mathbf{n}) \nabla_{\mathbf{x}} \mathbf{u}_{2} \mathbf{n}, \nabla_{\mathbf{n}}\left(f_{1}-f_{2}\right)^{2}\right) \\
& =\frac{1}{2}\left((2 \mathbf{n} \otimes \mathbf{n}-\mathrm{Id})\left(f_{1}-f_{2}\right)^{2}: \nabla_{\mathbf{x}} \mathbf{u}_{2}\right) \\
& \leq C\left\|f_{1}-f_{2}\right\|_{L^{4}\left(\Omega ; L^{2}(\mathbb{S})\right)}^{2}\left\|\nabla_{\mathbf{x}} \mathbf{u}_{2}\right\|_{L^{2}(\Omega)} \\
& \leq C\left[\left\|\nabla_{\mathbf{x}} f_{1}-\nabla_{\mathbf{x}} f_{2}\right\|_{L^{2}(\Omega \times \mathbb{S})}\left\|f_{1}-f_{2}\right\|_{L^{2}(\Omega \times \mathbb{S})}+\left\|f_{1}-f_{2}\right\|_{L^{2}(\Omega \times \mathbb{S})}^{2}\right] \\
& \leq \frac{1}{8}\left\|\nabla_{\mathbf{x}} f_{1}-\nabla_{\mathbf{x}} f_{2}\right\|_{L^{2}(\Omega \times \mathbb{S})}^{2}+C\left\|f_{1}-f_{2}\right\|_{L^{2}(\Omega \times \mathbb{S})}^{2} .
\end{aligned}
$$

One has from the Cauchy-Schwartz inequality that

$$
\begin{aligned}
Q_{3} & \leq C\left\|\nabla_{\mathbf{x}} f_{1}-\nabla_{\mathbf{x}} f_{2}\right\|_{L^{2}(\Omega \times \mathbb{S})}\left\|f_{1}-f_{2}\right\|_{L^{2}(\Omega \times \mathbb{S})} \\
& \leq \frac{1}{8}\left\|\nabla_{\mathbf{x}} f_{1}-\nabla_{\mathbf{x}} f_{2}\right\|_{L^{2}(\Omega \times \mathbb{S})}^{2}+C\left\|f_{1}-f_{2}\right\|_{L^{2}(\Omega \times \mathbb{S})}^{2} .
\end{aligned}
$$

Because Id $+\mathbf{n} \otimes \mathbf{n}$ is a positive definite matrix with smallest eigenvalue 1, we have

$$
Q_{4} \leq-\left\|\nabla_{\mathbf{x}} f_{1}-\nabla_{\mathbf{x}} f_{2}\right\|_{L^{2}(\Omega \times \mathbb{S})}^{2}
$$

Combining (4.8)-(4.14), we have for a.e. $t \in[0, T]$,

$$
\frac{d}{d t}\left\|f_{1}-f_{2}\right\|_{L^{2}(\Omega \times \mathbb{S})}^{2} \leq C\left(1+\left\|f_{1}\right\|_{H^{1}(\Omega \times \mathbb{S})}+\left\|f_{1}\right\|_{H^{1}(\Omega \times \mathbb{S})}^{2}\right)\left\|f_{1}-f_{2}\right\|_{L^{2}(\Omega \times \mathbb{S})}^{2} .
$$

Because (4.5) implies $\left\|f_{1}\right\|_{L^{2}\left(0, T ; H^{1}(\Omega \times \mathbb{S})\right)} \leq C$ and (4.4) yields $\left.\left(f_{1}-f_{2}\right)\right|_{t=0}=0$, it follows from Gronwall's inequality that $f_{1} \equiv f_{2}$ a.e. on $[0, T] \times \Omega \times \mathbb{S}$, and hence from (4.6) and the Poincaré inequality that $\mathbf{u}_{1} \equiv \mathbf{u}_{2}$ on a.e. $[0, T] \times \Omega \times \mathbb{S}$. This ends the proof of Theorem 4.1 .

\section{REFERENCES}

[1] H. Bae and K. Trivisa, On the Doi model for the suspensions of rod-like molecules: Global-intime existence, Commun. Math. Sci., 11, 831-850, 2013.

[2] J.W. Barrett and E. Süli, Existence and equilibration of global weak solutions to kinetic models for dilute polymers I: Finitely extensible nonlinear bead-spring chains, Math. Model. Meth. Appl. Sci., 21(6), 1211-1289, 2011.

[3] J.W. Barrett and E. Süli, Existence and equilibration of global weak solutions to kinetic models for dilute polymers II: Hookean-type bead-spring chains, Math. Model. Meth. Appl. Sci., 22(5), 1150024, 2012.

[4] G.K. Batchelor, Slender-body theory for particles of arbitrary cross-section in Stokes flow, J. Fluid Mech., 44, 419-440, 1970

[5] X.Q. Chen and J.G. Liu, Global weak entropy solution to Doi-Saintillan-Shelley model for active and passive rod-like particle suspensions, J. Diff. Eqs., 254, 2764-2802, 2013.

[6] P. Constantin, Nonlinear Fokker-Planck Navier-Stokes systems, Commun. Math. Sci., 3, 531544,2005

[7] P. Constantin and N. Masmoudi, Global well-posedness for a Smoluchowski equation coupled with Navier-Stokes equations in 2D, Commun. Math. Phys., 278, 179-191, 2008.

[8] P. Constantin and G. Seregin, Global Regularity of solutions of coupled Navier-Stokes equations and nonlinear Fokker-Planck equations, Disc. Cont. Dyn. Sys., 26, 1185-1196, 2010.

[9] M. Doi, Molecular-dynamics and rheological properties of concentrated solutions of rodlike polymers in isotropic and liquid-crystalline phases, J. Polym. Sci. Polym. Phys. Ed., 19, 229-243, 1981. 
[10] M. Doi and S.F. Edwards, The Theory of Polymer Dynamics, Oxford Univ. Press, Oxford, 1986.

[11] M. Dreher and A. Jüngel, Compact families of piecewise constant functions in $L^{p}(0, T ; B)$, Nonlin. Anal., 75, 3072-3077, 2012.

[12] J.A. Dubinskĭi, Weak convergence for nonlinear elliptic and parabolic equations, Mat. Sb., 67, 609-642, 1965.

[13] L.C. Evans, Partial Differential Equations, American Mathematical Society, Providence, Rhode Island, 1998.

[14] D. Gilbarg and N.S. Trudinger, Elliptic Partial Differential Equations of Second Order, Springer, Berlin, 2001.

[15] C. Helzel and F. Otto, Multiscale simulations for suspensions of rod-like molecules, J. Comput. Phys., 216(1), 52-75, 2006.

[16] C. Helzel, F. Otto, and A.E. Tzavaras, A kinetic model for the sedimentation of rod-like particles, 2011, preprint.

[17] G.B. Jefferym, The motion of ellipsoidal particles immersed in a viscous fluid, Proc. R. Soc. London, Ser. A., 102, 161-179, 1922.

[18] J. Kačur, Method of Rothe in Evolution Equations, Teubner Texte zur Mathematik B.G. Teubner, Leipzig, 80, 1985.

[19] S. Kim and S.J. Karrila, Microhydrodynamics: Principles and selected applications, Boston: Butterworth-Heinemann, 1991.

[20] P. L. Lions and N. Masmoudi, Global solutions of weak solutions to some micromacro models, C.R. Math. Sci. Paris., 345, 15-20, 2007.

[21] F. Otto and A.E. Tzavaras, Continuity of velocity gradient in suspensions of rod-like molecules, Commun. Math. Phys., 277, 729-758, 2008.

[22] E. Rothe, Zweidimensionale parabolische Randwertaufgaben als Grenzfall eindimensionaler Randwertaufgaben, Math. Ann., 102, 650-670, 1930.

[23] D. Saintillan and M.J. Shelley, Instabilities, pattern formation, and mixing in active suspensions, Phys. Fluids, 20, 123304, 2008.

[24] J. Simon, Compact sets in the space $L^{p}(0, T ; B)$, Ann. Mat. Pura Appl., 146, 65-96, 1987.

[25] Y.Z. Sun and Z.F. Zhang, Global well-posedness for the $2 D$ micro-macro models in the bounded domain, Commun. Math. Phys., 303, 361-383, 2011.

[26] R. Teman, Navier-Stokes Equations. Theory and Numerical Analysis (Third Edition), NorthHolland, Amsterdam, 1984. 